\title{
Transmetalation as key step in the Diastereo- and Enantioselective Synergistic Cu/Pd-catalyzed allylboration of alkynes with racemic allylic carbonates
}

Jaime Mateos, Noelia Fuentes-Vara, Laura Fra, Eva Rivera-Chao, Nuria VázquezGaliñanes, Andrea Chaves-Pouso and Martín Fañanás-Mastral*

Centro Singular de Investigación en Química Biolóxica e Materiais Moleculares (CiQUS), Departamento de Química Orgánica, Universidade de Santiago de Compostela, Jenaro de la Fuente s/n, 15782 Santiago de Compostela, Spain. 


\section{Table of contents}

1. General methods $\quad$ S3

2. List of starting materials $\quad$ S4

3. Table S1. Screening of chiral LPd ligands S5

4. General procedures for the diastereo- and enantioselective $\mathrm{Cu} / \mathrm{Pd}$-catalyzed S6 allylboration of alkynes

$\begin{array}{ll}\text { 5. Compound characterization } & \text { S7 }\end{array}$

6 Determination of enantiomeric ratios $\quad$ S10

$\begin{array}{lll}\text { 7. } & { }^{1} \mathrm{H}-\mathrm{NMR} \text { and }{ }^{13} \mathrm{C}-\mathrm{NMR} \text { spectra } & \text { S16 }\end{array}$

8. Stereochemical determination of compounds trans-4a and cis-4d by NOE S26 experiments

9. References 


\section{General methods}

- All reactions were performed under argon atmosphere using oven dried glassware and using standard Schlenk techniques. Solvents were dried using an MBraun SPS 800 system. All chemicals and copper complexes were purchased from Acros Organics Ltd., Aldrich Chemical Co. Ltd., Alfa Aesar, Apollo, Strem Chemicals Inc., Fluorochem Ltd. or TCI Europe N.V. chemical companies and used without further purification, unless otherwise noted.

- Analytical thin layer chromatography was carried out on silica-coated aluminium plates (silica gel 60 F254 Merck) and components were visualized by UV light, 12 and $\mathrm{KMnO}_{4}$ staining. Flash column chromatography was performed on silica gel 60 (Merck, 230-400 mesh) without previous deactivation, unless otherwise stated.

- GC-MS analyses were performed in an Agilent instrument GC-6890N equipped with Chemical Ionization (CI) MS-5973 detector.

- High Resolution Mass spectrometry was carried out on a Bruker microTOF spectrometer using $\mathrm{Cl}$ or $\mathrm{APCl}$.

- ${ }^{1} \mathrm{H}$ - and ${ }^{13} \mathrm{C}-\mathrm{NMR}$ experiments were carried out using a Varian Inova $500 \mathrm{MHz}$ or a Varian Mercury $300 \mathrm{MHz}$ NMR spectrometers. Chemical shift values are reported in ppm with the solvent resonance as the internal standard $\left(\mathrm{CHCl}_{3}: \delta 7.26\right.$ for ${ }^{1} \mathrm{H}, \delta 77.16$ for $\left.{ }^{13} \mathrm{C}\right)$. Coupling constants $(J)$ are given in Hertz $(\mathrm{Hz})$. Multiplicities are reported as follows: $\mathrm{s}=$ singlet, $\mathrm{d}=$ doublet, $\mathrm{t}=$ triplet $\mathrm{q}=$ quartet, $\mathrm{p}=$ pentet, $\mathrm{m}=$ multiplet or as a combination of them .

- Enantiomeric ratios were determined by high pressure liquid chromatography, HPLC analysis Agilent HPLC 1100 Series and Hitachi Elite LaChrom DAD, FL.

- Optical rotation was determined in a Jasco P-2000 Polarimeter.

- In order to preclude side hydroboration reactions, commercial alkynes and $\mathrm{B}_{2} \mathrm{pin}_{2}$ were dried over $\mathrm{Na}_{2} \mathrm{SO}_{4}$ prior to being used. 


\section{List of starting materials}

Alkynes $\mathbf{1} \mathbf{a}-\mathbf{e}$ and $\mathrm{B}_{2}(\mathrm{pin})_{2}$ were obtained from commercial sources. Allyl derivates $\mathbf{2} \mathbf{a}-\mathbf{d}^{[1]}, \mathbf{2} \mathbf{e}^{[2]}$ $\mathbf{2 f}^{[1]}$ and $\mathbf{5}^{[1]}$ were prepared according to described procedures. Phosphines used to make copper catalysts $\left(\mathrm{L}_{\mathrm{Cu}}\right)$ and ligands shown in Table S1 were purchased from commercial sources. Ligands $\mathbf{L} \mathbf{2}, \mathbf{L} \mathbf{5}-\mathbf{7}^{[3]} \mathbf{L} \mathbf{3}^{[4]}$ and $\mathbf{L} \mathbf{4}^{[5]}$ were prepared according to literature procedures.

- Alkynes

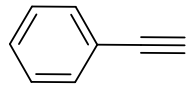

$1 \mathrm{a}$<smiles>C#CS</smiles>

$1 \mathrm{~b}$
$n-\mathrm{Bu}=$

1c

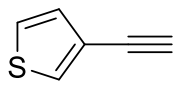

1d

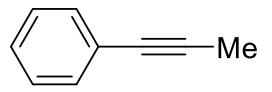

$1 e$

- Allylic substrates<smiles>COC(OC)O[C@H]1C=CCC(C(C)=O)C1</smiles>

$2 a$

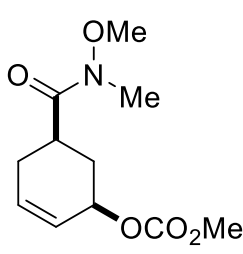

$2 b$

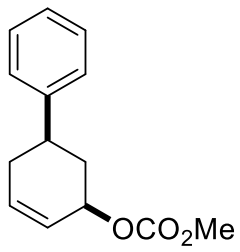

2c

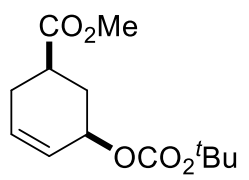

2d<smiles>CCOCOC1C=CCC(C(C)=O)C1</smiles>

$2 e$<smiles>CC(=O)OC1C=CCC(C(C)=O)C1</smiles>

$2 f$<smiles>CC(=O)C1CC=C[C@H](Cl)C1</smiles> 


\section{Table S1. Screening of chiral Lpd ligands}

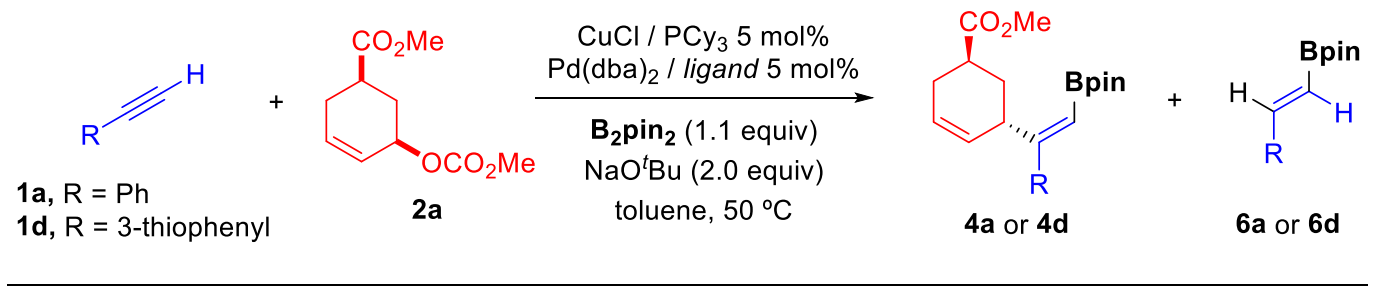

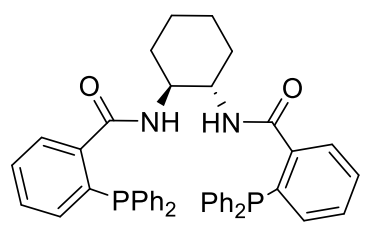

$(S, S)-D A C H-P h-T r o s t$ only $6^{*}$<smiles>Pc1ccccc1P</smiles>

(S)-Ph-Phox 4a, $12 \%$ / 6a, $30 \%$<smiles></smiles>

(S)- ${ }^{t} \mathrm{Bu}-\mathrm{Phox}$

4a, $17 \%$ / 6a, $18 \%$ 4d, $11 \% / 6 d, 20 \%$

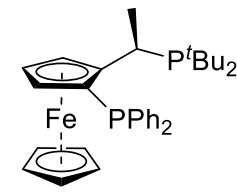

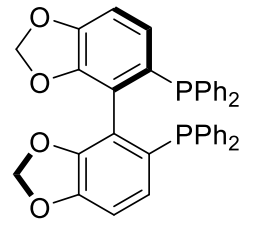

$(R)$-SEGPHOS only $\mathbf{6}^{*}$<smiles>C[C@H](c1ccccc1)N([C@H](C)c1ccccc1)P(Oc1ccc2ccccc2c1-c1c(O)ccc2ccccc12)c1ccccc1</smiles>

$(S, R, R)$-Feringa ligand

4a, $15 \%$ / 6a, $20 \%$

4d, $5 \% / 6$ d, $30 \%$

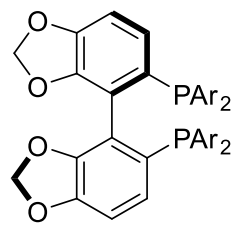

(R)-DTBM-SEGPHOS

$\mathrm{Ar}=3,5-{ }^{t} \mathrm{Bu}-4-\mathrm{OMe}-\mathrm{C}_{6} \mathrm{H}_{2}$ only $\mathbf{6}^{*}$<smiles>c1ccc(-c2ccc3ccccc3c2-c2c(-c3ccccc3)ccc3ccccc23)cc1</smiles>

(S)-BINAP only $6^{*}$<smiles>CC(C)P(C)[Pb](C)c1nc2ccccc2nc1P(C)C(C)(C)C</smiles>

$(R, R)$-QuinoxP* only $6^{*}$<smiles>COc1cc(OC)c(-c2c(OC)cc(OC)cc2[N+](=O)[O-])c([N+](=O)[O-])c1</smiles>

(R)-DTBM-GARPHOS only $6^{*}$<smiles>CC(C)(C)P1Cc2ccccc2[C@@H]1[C@@H]1c2ccccc2C[P@@]1C(C)(C)C</smiles>

(1S, 1'S,2R,2'R)-DuanPhos only $6^{*}$<smiles>PC([PH2+]c1ccccc1)c1ccccc1</smiles>

$(S, S)-P h-B P E$ only $6^{*}$<smiles>COc1ccc2ccccc2c1-c1c(-c2ccccc2)ccc2ccccc12</smiles>

(R)-MOP only $\mathbf{6}^{*}$

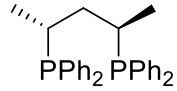

$(R, R)$-BDPP only $6^{*}$<smiles>C[C@@H]1CC[C@@H](C)P1c1ccccc1P1[C@H](C)CC[C@H]1C</smiles>

(S,S)-Me-DuPhos 4d, $7 \% / 6 d, 28 \%$

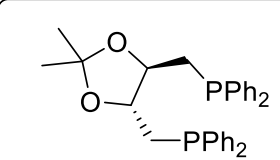

$(R, R)$-DIOP (L1)

4a, $87 \%$ / 6a, $10 \%$ 4d, $92 \% / 6 d, 8 \%$

${ }^{*} \mathrm{~B}_{2} \mathrm{pin}_{2}$ allylation products were detected in the crude NMR although these products were unstable upon purification and could not be isolated 


\section{General procedures}

Method A: General procedure for the diastereoselective Cu/Pd-catalyzed allylboration of alkynes.

$\mathrm{CuCl}(5 \mathrm{~mol} \%, 0.01 \mathrm{mmol})$ and $\mathrm{PCy}_{3}(5 \mathrm{~mol} \%, 0.01 \mathrm{mmol})$ were dissolved in dry toluene $(0.5$ $\mathrm{mL}$ ), stirred during 15 minutes at room temperature and added to the reaction vial (using 0.5 $\mathrm{mL}$ of toluene for washing) containing $\mathrm{B}_{2} \mathrm{pin}_{2}\left(1.1\right.$ equiv, $0.22 \mathrm{mmol}$ ) and $\mathrm{NaO}{ }^{t} \mathrm{Bu}$ (2.0 equiv, 0.4 $\mathrm{mmol}$ ) to afford a dark brown suspension. In a separate vial, $\mathrm{Pd}(\mathrm{dba})_{2}(5 \mathrm{~mol} \%, 0.01 \mathrm{mmol})$ and dppf ( $5 \mathrm{~mol} \%, 0.01 \mathrm{mmol}$ ) were dissolved in $0.5 \mathrm{~mL}$ of dry toluene and stirred 15 minutes at rt. Then, the corresponding alkyne (1.0 equiv, $0.2 \mathrm{mmol})$ and racemic allylic substrate (1.2 equiv, $0.24 \mathrm{mmol}$ ) were added to this vial and this solution was finally added to the reaction vial and heated at $50{ }^{\circ} \mathrm{C}$ for the indicated time. After this time, the reaction was quenched by addition of saturated aqueous solution of $\mathrm{NH}_{4} \mathrm{Cl}(3 \times 10 \mathrm{~mL})$ and extracted with $\mathrm{Et}_{2} \mathrm{O}(15 \mathrm{~mL})$. The organic layer was dried over $\mathrm{Na}_{2} \mathrm{SO}_{4}$, filtered and solvent was removed under vacuum. The final product was purified by $\mathrm{SiO}_{2}$ column chromatography using the indicated mixture of eluents for each case.

Method B: General procedure for the enantioselective $\mathrm{Cu} / \mathrm{Pd}$-catalyzed allylboration of alkynes.

$\mathrm{CuCl}(5 \mathrm{~mol} \%, 0.01 \mathrm{mmol}$ ) and rac-Tol-BINAP ( $5 \mathrm{~mol} \%, 0.01 \mathrm{mmol}$ ) were dissolved in dry DMA $(0.5 \mathrm{~mL}$ ), stirred during 15 minutes at $\mathrm{rt}$ and added to the reaction vial (using $0.5 \mathrm{~mL}$ of DMA for washing) which contains $\mathrm{B}_{2} \mathrm{pin}_{2}$ (1.1 equiv, $0.22 \mathrm{mmol}$ ) and $\mathrm{NaO}^{t} \mathrm{Bu}$ (2.0 equiv, $0.4 \mathrm{mmol}$ ) to afford a dark brown suspension. In a separate vial, $\mathrm{Pd}(\mathrm{dba})_{2}(5 \mathrm{~mol} \%, 0.01 \mathrm{mmol}$ ) and DIOPderivative ( $5 \mathrm{~mol} \%, 0.01 \mathrm{mmol}$ ) in $0.5 \mathrm{~mL}$ of DMA were added and stirred 15 minutes at $\mathrm{rt}$. Then, the corresponding alkyne (1.0 equiv, $0.2 \mathrm{mmol}$ ) and allylic carbonate (1.2 equiv, 0.24 mmol) were added to this vial and this solution was finally added to the reaction vial and stirred at $30^{\circ} \mathrm{C}$ for the indicated time. After this time, the reaction was quenched by addition of saturated aqueous solution of $\mathrm{NH}_{4} \mathrm{Cl}(3 \times 10 \mathrm{~mL})$ and extracted with $\mathrm{Et}_{2} \mathrm{O}(15 \mathrm{~mL})$. The organic layer was dried over $\mathrm{Na}_{2} \mathrm{SO}_{4}$, filtered and solvent was removed under vacuum. The final product was purified by silica gel column chromatography using (10:1 Pentane: $\mathrm{Et}_{2} \mathrm{O}$ mixture as eluent). 


\section{Compound characterization}

Methyl $\left(1 R^{*}, 5 S^{*}\right)-5-((E)-1-p h e n y l-2-(4,4,5,5-t e t r a m e t h y l-1,3,2-d i o x a b o r o l a n-2-$

yl)vinyl)cyclohex-3-ene-1-carboxylate (4a)

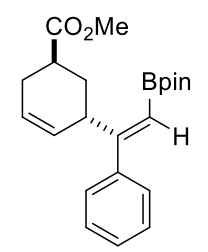

Obtained from $\mathbf{1 a}, \mathbf{2} \mathbf{a}$ and $\mathrm{B}_{2}(\mathrm{pin})_{2}$ as a yellow oil after column chromatography

(hexane/EtOAc 6:1) following general procedure A in 95\% yield. ${ }^{1} \mathbf{H}$ NMR (500 $\left.\mathrm{MHz}, \mathrm{CDCl}_{3}\right): \delta 7.25-7.22(\mathrm{~m}, 2 \mathrm{H}), 7.19-7.16(\mathrm{~m}, 3 \mathrm{H}), 5.71(\mathrm{~s}, 2 \mathrm{H}), 5.39(\mathrm{~s}, 1 \mathrm{H})$, $4.09-3.98(\mathrm{~m}, 1 \mathrm{H}), 3.59(\mathrm{~s}, 3 \mathrm{H}), 2.49-2.43(\mathrm{~m}, 1 \mathrm{H}), 2.29-2.19(\mathrm{~m}, 1 \mathrm{H}), 2.12-$ $2.05(\mathrm{~m}, 1 \mathrm{H}), 1.95(\mathrm{dt}, J=13.4,6.7 \mathrm{~Hz}, 1 \mathrm{H}), 1.76$ (ddd, $J=13.4,7.5,3.9 \mathrm{~Hz}, 1 \mathrm{H}), 1.22(\mathrm{~s}, 12 \mathrm{H})$. ${ }^{13} \mathrm{C}$ NMR $\left(126 \mathrm{MHz} \mathrm{CDCl}_{3}\right): \delta 175.9,164.7,144.1,130.6,127.9,127.5,127.3,126.3,83.3,51.7$, 39.1, 36.7, 29.9, 26.5, 25.2, 25.1, 25.0. ${ }^{11}$ B NMR (160 MHz, $\left.\mathrm{CDCl}_{3}\right): \delta 22.50$. HRMS (Cl) m/z: Calc. for $\mathrm{C}_{22} \mathrm{H}_{29} \mathrm{BO}_{4}\left[\mathrm{M}^{+}\right]$368.2159, found 368.2159.

\section{Methyl $\left(1 R^{*}, 5 S^{*}\right)-5-((E)-2-(4,4,5,5-$ tetramethyl-1,3,2-dioxaborolan-2-yl)-1-}

(trimethylsilyl)vinyl)cyclohex-3-ene-1-carboxylate (4b)

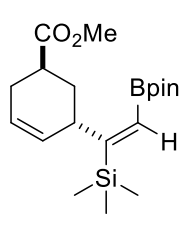

Obtained from $\mathbf{1 b}, \mathbf{2}$ a and $\mathrm{B}_{2}(\text { pin })_{2}$ as a yellow oil after column chromatography (hexane/EtOAc) following general procedure $A$ in $98 \%$ yield. ${ }^{1} \mathbf{H}$ NMR $(500 \mathrm{MHz}$, $\left.\mathrm{CDCl}_{3}\right): \delta 5.91(\mathrm{~s}, 1 \mathrm{H}), 5.75-5.70(\mathrm{~m}, 1 \mathrm{H}), 5.59-5.55(\mathrm{~m}, 1 \mathrm{H}), 3.69(\mathrm{~s}, 3 \mathrm{H}), 3.62$ (ddt, $J=8.9,5.9,2.9,1 \mathrm{H}), 2.83(\mathrm{ddd}, J=9.7,5.7,4.1 \mathrm{~Hz}, 1 \mathrm{H}), 2.46-2.39(\mathrm{~m}, 1 \mathrm{H})$, $2.21(\mathrm{ddq}, J=17.7,6.0,2.9 \mathrm{~Hz}, 1 \mathrm{H}$ ), $2.08(\mathrm{dt}, J=12.1,5.8 \mathrm{~Hz}, 1 \mathrm{H}), 1.81$ (ddd, $J=13.3,9.2,4.1$ $\mathrm{Hz}, 1 \mathrm{H}), 1.26(\mathrm{~s}, 12 \mathrm{H}), 0.10(\mathrm{~s}, 9 \mathrm{H}) .{ }^{13} \mathrm{C}$ NMR $\left(126 \mathrm{MHz}, \mathrm{CDCl}_{3}\right): \delta 175.8,170.8,130.9,125.9$, $83.3,51.8,39.9,37.3,31.3,26.3,25.1,25.0 .0 .2 .{ }^{11}$ B NMR $\left(160 \mathrm{MHz}, \mathrm{CDCl}_{3}\right): \delta$ 29.26. HRMS (APCI) m/z: Calc. For $\mathrm{C}_{19} \mathrm{H}_{33} \mathrm{BO}_{4} \mathrm{Si}[\mathrm{M}+\mathrm{H}]$ 365.2314, found 365.2318.

\section{Methyl (1 $\left.R^{*}, 5 S^{*}\right)-5-((Z)-1-(4,4,5,5$-tetramethyl-1,3,2-dioxaborolan-2-yl)hex-1-en-2-}

yl)cyclohex-3-ene-1-carboxylate (4c)

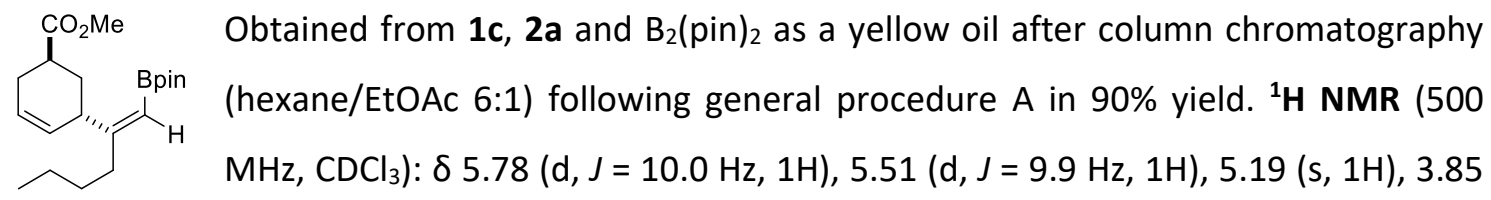
(s, $1 \mathrm{H}), 3.70(\mathrm{~s}, 3 \mathrm{H}), 2.82-2.75(\mathrm{~m}, 1 \mathrm{H}), 2.38(\mathrm{~d}, J=17.6 \mathrm{~Hz}, 1 \mathrm{H}), 2.23(\mathrm{~d}, J=17.4 \mathrm{~Hz}, 1 \mathrm{H}), 2.07-$ $2.02(\mathrm{~m}, 3 \mathrm{H}), 1.79$ (ddd, $J=12.6,7.2,4.1 \mathrm{~Hz}, 1 \mathrm{H}), 1.42(\mathrm{q}, J=14.9,7.6 \mathrm{~Hz} 2 \mathrm{H}), 1.35-1.27(\mathrm{~m}$, 3H), $1.25(\mathrm{~s}, 12 \mathrm{H}), 0.88(\mathrm{t}, J=7.2 \mathrm{~Hz}, 3 \mathrm{H}) .{ }^{13} \mathrm{C}$ NMR $\left(126 \mathrm{MHz}, \mathrm{CDCl}_{3}\right): \delta 176.0,168.4,130.1$, $125.9,82.9,51.8,39.1,37.4,34.7,30.9,30.7,26.4,24.9,24.8,22.8,14.2 .{ }^{11}$ B NMR (160 MHz, $\left.\mathrm{CDCl}_{3}\right): \delta$ 30.02. HRMS (APCI) m/z: Calc. for $\mathrm{C}_{20} \mathrm{H}_{34} \mathrm{BO}_{4}[\mathrm{M}+\mathrm{H}] 349.2545$, found 349.2549 . 
Methyl $\left(1 R^{*}, 5 S^{*}\right)-5-((E)-2-(4,4,5,5-t e t r a m e t h y l-1,3,2-$ dioxaborolan-2-yl)-1-(thiophen-3-

yl)vinyl)cyclohex-3-ene-1-carboxylate (4d)

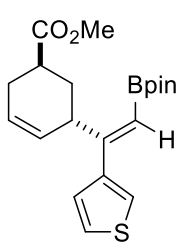

Obtained from $\mathbf{1 d}, \mathbf{2 a}$ and $\mathrm{B}_{2}(\text { pin })_{2}$ as a yellow oil after column chromatography

(hexane/Et ${ }_{2} \mathrm{O} 5: 1$ ) following general procedure $\mathrm{A}$ in $90 \%$ yield. ${ }^{1} \mathrm{H}$ NMR (300 $\left.\mathrm{MHz}, \mathrm{CDCl}_{3}\right): \delta 7.37$ - $7.36(\mathrm{~m}, 1 \mathrm{H}), 7.22$ - $7.17(\mathrm{~m}, 2 \mathrm{H}), 5.86-5.80(\mathrm{~m}, 1 \mathrm{H}), 5.79-$

$5.75(\mathrm{~m}, 1 \mathrm{H}), 5.66(\mathrm{~s}, 1 \mathrm{H}), 4.12-4.06(\mathrm{~m}, 1 \mathrm{H}), 3.69(\mathrm{~s}, 3 \mathrm{H}), 2.76-2.71(\mathrm{~m}, 1 \mathrm{H})$, $2.46-2.40(\mathrm{~m}, 1 \mathrm{H}), 2.28-2.22(\mathrm{~m}, 1 \mathrm{H}), 2.14-2.08(\mathrm{~m}, 1 \mathrm{H}), 1.99-1.92(\mathrm{~m}, 1 \mathrm{H}), 1.28(\mathrm{~s}, 12 \mathrm{H})$. ${ }^{13} \mathrm{C} \mathrm{NMR}\left(75 \mathrm{MHz}, \mathrm{CDCl}_{3}\right): \delta 175.8,158.5,143.9,130.9,127.6,126.2,124.7,122.2,83.3,52.8$, 38.7, 37.2, 30.1, 26.3, 25.02, 24.7. ${ }^{11}$ B NMR (300 MHz, CDCl $)$ : $\delta$ 30.16. HRMS (APCI) m/z: Calc. for $\mathrm{C}_{20} \mathrm{H}_{28} \mathrm{BO}_{4} \mathrm{~S}[\mathrm{M}+\mathrm{H}] 375.1796$, found 375.1800 .

Methyl (1 $\left.R^{*}, 5 S^{*}\right)-5-((Z)-1-$ phenyl-2-(4,4,5,5-tetramethyl-1,3,2-dioxaborolan-2-yl)prop-1-en1-yl)cyclohex-3-ene-1-carboxylate (4e)

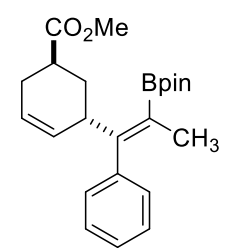

Obtained from $1 \mathbf{e}, \mathbf{2 a}$ and $\mathrm{B}_{2}(\mathrm{pin})_{2}$ as a yellow oil after column chromatography (hexane/EtOAc 6:1) following general procedure A in $70 \%$ yield. ${ }^{1} \mathrm{H}$ NMR $\left(500 \mathrm{MHz}, \mathrm{CDCl}_{3}\right): \delta 7.30-7.20(\mathrm{~m}, 3 \mathrm{H}), 7.00-6.97(\mathrm{~m}, 2 \mathrm{H}), 5.69$ $(\mathrm{s}, 2 \mathrm{H}), 3.88-3.83(\mathrm{~m}, 1 \mathrm{H}), 3.62(\mathrm{~s}, 3 \mathrm{H}), 2.17-2.10(\mathrm{~m}, 2 \mathrm{H}), 1.99-1.88(\mathrm{~m}$, 2H), 1.74 (ddd, $J=13.1,9.4,2.2 \mathrm{~Hz}, 1 \mathrm{H}), 1.45$ (s, 3H), 1.31 (s, 12H). ${ }^{13} \mathrm{C}$ NMR (126 MHz, CDCl $)$ : $\delta 176.1,155.7,141.1,130.3,128.7,127.8,126.3,126.0,83.3,51.5,40.3,36.0,29.6,26.2$, 24.9, 24.8, 18.2. ${ }^{11}$ B NMR (160 MHz, $\left.\mathrm{CDCl}_{3}\right): \delta$ 31.00. HRMS (APCl) m/z: Calc. for $\mathrm{C}_{23} \mathrm{H}_{32} \mathrm{BO}_{4}[\mathrm{M}+\mathrm{H}]$ 383.2388 , found 383.2395 .

$\left(1 R^{*}, 5 S^{*}\right)-N$-methoxy-N-methyl-5-((E)-1-phenyl-2-(4,4,5,5-tetramethyl-1,3,2-dioxaborolan-2yl)vinyl)cyclohex-3-enecarboxamide (4f)

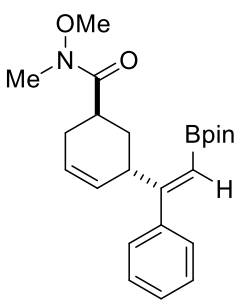

Obtained from $\mathbf{1 a}, \mathbf{2} \mathbf{b}$ and $\mathrm{B}_{2}(\text { pin })_{2}$ as a yellow oil after column chromatography (hexane/EtOAc 6:1) following general procedure A in 97\% yield. ${ }^{1} \mathbf{H}$ NMR $\left(300 \mathrm{MHz}, \mathrm{CDCl}_{3}\right) \delta 7.35-7.30(\mathrm{~m}, 2 \mathrm{H}), 7.27-7.21(\mathrm{~m}, 3 \mathrm{H})$, $5.94-5.86(\mathrm{~m}, 2 \mathrm{H}), 5.37(\mathrm{~s}, 1 \mathrm{H}), 4.12(\mathrm{ddt}, J=7.0,5.1,2.4 \mathrm{~Hz}, 1 \mathrm{H}), 3.31(\mathrm{~s}$, $3 \mathrm{H}), 3.06(\mathrm{~s}, 3 \mathrm{H}), 2.63(\mathrm{bs}, 1 \mathrm{H}) 2.17(\mathrm{dd}, J=9.3,2.6 \mathrm{~Hz}, 1 \mathrm{H}), 2.08(\mathrm{t}, J=7.1$ $\mathrm{Hz}, 1 \mathrm{H}), 1.96$ (ddd, $J=13.7,11.4,7.2 \mathrm{~Hz}, 1 \mathrm{H}), 1.75(\mathrm{~s}, 1 \mathrm{H}), 1.28(\mathrm{~d}, J=2.5 \mathrm{~Hz}, 12 \mathrm{H}) .{ }^{13} \mathrm{C}$ NMR $(75$ $\left.\mathrm{MHz}, \mathrm{CDCl}_{3}\right) \delta 164.7,145.0,129.5,128.1,127.9,127.8,127.3,83.4,61.6,39.5,32.4,30.0,27.3$, 25.20, 25.16. ${ }^{11}$ B NMR (160 MHz, $\mathrm{CDCl}_{3}$ ): $\delta$ 29.91. HRMS (APCI) m/z: Calc. for $\mathrm{C}_{23} \mathrm{H}_{33} \mathrm{BNO}_{4}$ $[\mathrm{M}+\mathrm{H}]:$ 398.2497, found 398.2501. 
4,4,5,5-Tetramethyl-2-((E)-2-((1R*,3S*)-1,2,3,6-tetrahydro-[1,1'-biphenyl]-3-yl)-2-(thiophen3-yl)vinyl)-1,3,2-dioxaborolane (4g)

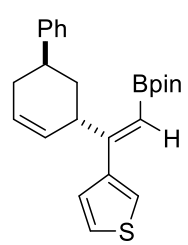

Obtained from $\mathbf{1 d}, \mathbf{2 c}$ and $\mathrm{B}_{2}(\mathrm{pin})_{2}$ as a yellow oil after column chromatography (hexane/Et $\mathrm{E}_{2} \mathrm{O} 10: 1$ ) following general procedure $\mathrm{A}$ in $81 \%$ yield. The presence of protoboration side product was detected in a $10 \%$ and could not be separated by column cromatography. ${ }^{1} \mathrm{H}$ NMR $\left(300 \mathrm{MHz}, \mathrm{CDCl}_{3}\right) \delta 7.39(\mathrm{dd}, J=2.8,1.4 \mathrm{~Hz}$, 1H), $7.30-7.17(\mathrm{~m}, 7 \mathrm{H}), 5.99(\mathrm{~d}, J=9.3 \mathrm{~Hz}, 1 \mathrm{H}), 5.85(\mathrm{~d}, J=10.6 \mathrm{~Hz}, 1 \mathrm{H}), 5.61(\mathrm{~d}, J=1.4 \mathrm{~Hz}$, 1H), $3.97-3.90(\mathrm{~m}, 1 \mathrm{H}), 3.05(\mathrm{p}, J=5.8 \mathrm{~Hz}, 1 \mathrm{H}), 2.48(\mathrm{~d}, J=18.8 \mathrm{~Hz}, 1 \mathrm{H}), 2.31(\mathrm{~d}, J=18.6 \mathrm{~Hz}$, 1H), $2.15-2.04(\mathrm{~m}, 1 \mathrm{H}), 1.92(\mathrm{dt}, J=12.7,6.2 \mathrm{~Hz}, 1 \mathrm{H}), 1.14(\mathrm{~s}, 6 \mathrm{H}), 1.10(\mathrm{~s}, 6 \mathrm{H}) .{ }^{13} \mathrm{C}$ NMR $(126$ $\left.\mathrm{MHz}, \mathrm{CDCl}_{3}\right): \delta 159.2,146.3,144.0,143.1,131.7,128.1,127.7,127.3,126.9,125.8,124.3$, $122.2,82.9,38.2,36.6,35.2,29.9,24.8,24.7 .{ }^{11} \mathrm{~B}$ NMR (160 MHz, $\left.\mathrm{CDCl}_{3}\right): \delta$ 30.39. HRMS (APCI) $\mathrm{m} / \mathrm{z}$ : Calc. for $\mathrm{C}_{24} \mathrm{H}_{30} \mathrm{BO}_{2} \mathrm{~S}[\mathrm{M}+\mathrm{H}]$ 393.2054, found 393.2055.

\section{4,4,5,5-Tetramethyl-2-((E)-2-phenyl-2-((1R*,3S*)-1,2,3,6-tetrahydro-[1,1'-biphenyl]-3-} yl)vinyl)-1,3,2-dioxaborolane (4h)

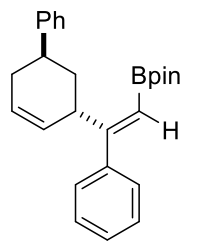

Obtained from $1 \mathrm{a}, \mathbf{2 c}$ and $\mathrm{B}_{2}(\mathrm{pin})_{2}$ as a yellow oil after column chromatography (hexane/EtOAc 6:1) following general procedure A in $98 \%$ yield. ${ }^{1}$ H NMR (500 $\left.\mathrm{MHz}, \mathrm{CDCl}_{3}\right): \delta 7.37$ - $7.34(\mathrm{~m}, 2 \mathrm{H}), 7.31-7.27(\mathrm{~m}, 5 \mathrm{H}), 7.21-7.14(\mathrm{~m}, 3 \mathrm{H}), 6.00$ $5.96(\mathrm{~m}, 1 \mathrm{H}), 5.89(\mathrm{dq}, J=10.1,2.3 \mathrm{~Hz}, 1 \mathrm{H}), 5.43(\mathrm{~s}, 1 \mathrm{H}), 4.00(\mathrm{tq}, J=6.2,2.9 \mathrm{~Hz}$, $1 \mathrm{H}), 2.81-2.76(\mathrm{~m}, 1 \mathrm{H}), 2.39-2.31(\mathrm{~m}, 1 \mathrm{H}), 2.29-2.21(\mathrm{~m}, 1 \mathrm{H}), 1.95-1.91(\mathrm{~m}, 2 \mathrm{H}), 1.24(\mathrm{~s}$, $6 \mathrm{H}), 1.21$ (s, 6H). ${ }^{13} \mathrm{C}$ NMR $\left(126 \mathrm{MHz}, \mathrm{CDCl}_{3}\right): \delta 165.7,146.8,144.6,131.3,128.4,127.9,127.8$, 127.6, 127.4, 127.3, 126.0, 83.3, 39.3, 36.4, 35.3, 30.7, 25.02, 25.00. ${ }^{11}$ B NMR (160 MHz, $\mathrm{CDCl}_{3}$ ): $\delta$ 29.87. HRMS (APCI) m/z: Calc. for $\mathrm{C}_{26} \mathrm{H}_{32} \mathrm{BO}_{2}[\mathrm{M}+\mathrm{H}]$ 387.2490, found 387.2496.

$\left(1 R^{*}, 5 R^{*}\right)$-Methyl 5-((E)-2-(4,4,5,5-tetramethyl-1,3,2-dioxaborolan-2-yl)-1-(thiophen-3yl)vinyl)cyclohex-3-enecarboxylate (cis-4d)

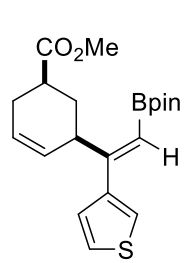

Obtained from $\mathbf{1 d}, \mathbf{5}$ and $\mathrm{B}_{2}(\mathrm{pin})_{2}$ as a yellow oil after column chromatography (hexane/Et ${ }_{2} \mathrm{O}$ 10:1) following the general procedure $A$ in $88 \%$ yield. ${ }^{1} \mathbf{H}$ NMR (500 MHz, $\left.\mathrm{CDCl}_{3}\right) \delta 7.43-7.41(\mathrm{~m}, 1 \mathrm{H}), 7.23$ (d, $\left.J=5.1 \mathrm{~Hz}, 1 \mathrm{H}\right), 7.19$ (dd, $J=5.0$, $2.8 \mathrm{~Hz}, 1 \mathrm{H}), 5.82-5.74(\mathrm{~m}, 2 \mathrm{H}), 5.73(\mathrm{~s}, 1 \mathrm{H}), 4.32-4.26(\mathrm{~s}, 1 \mathrm{H}), 3.68(\mathrm{~s}, 3 \mathrm{H})$, $2.78(\mathrm{ddq}, J=13.5,8.8,2.9 \mathrm{~Hz}, 1 \mathrm{H}), 2.40-2.30(\mathrm{~m}, 2 \mathrm{H}), 2.12-2.07(\mathrm{~m}, 1 \mathrm{H}), 1.89(\mathrm{q}, J=12.4 \mathrm{~Hz}$, 1H), 1.30 (s, 12H). ${ }^{13} \mathrm{C}$ NMR (126 MHz, $\left.\mathrm{CDCl}_{3}\right) \delta 176.3,159.2,143.2,132.1,127.6,125.3,124.8$, 122.9, 83.4, 51.9, 42.1, 40.3, 31.9, 27.7, 25.15, 25.07. ${ }^{11} \mathrm{~B}$ NMR $\left(160 \mathrm{MHz}, \mathrm{CDCl}_{3}\right): \delta 30.1$. HRMS (APCI) m/z: Calc. for $\mathrm{C}_{20} \mathrm{H}_{28} \mathrm{BO}_{4} \mathrm{~S}[\mathrm{M}+\mathrm{H}] 375.1796$, found 375.1799 . 


\section{Determination of enantiomeric ratios}

Methyl (1R,5S)-5-((E)-2-(4,4,5,5-tetramethyl-1,3,2-dioxaborolan-2-yl)-1-(thiophen-3-

yl)vinyl)cyclohex-3-ene-1-carboxylate (4d)

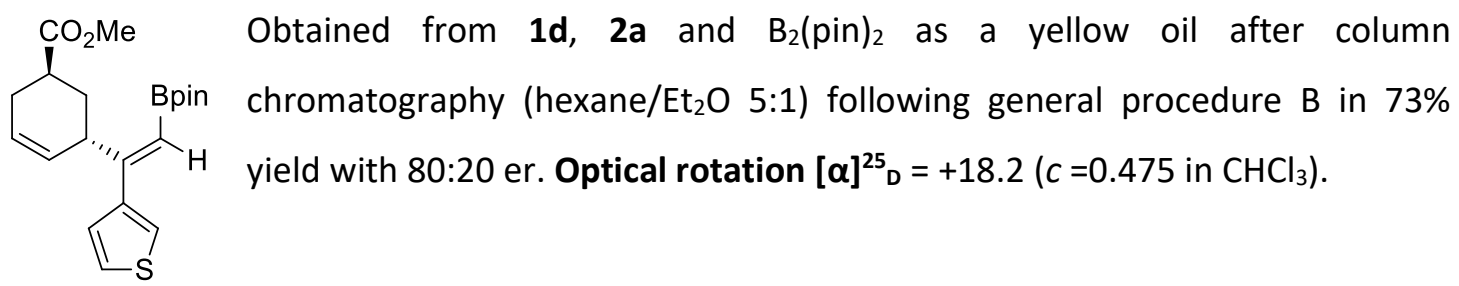

Enantiomeric purity was determined by chiral HPLC analysis [Cellulose1, $\mathrm{T}_{\text {oven }}=40^{\circ} \mathrm{C}$, flow $=$ $0.5 \mathrm{ml} / \mathrm{min}, \mathrm{Hex} / \mathrm{IPA} 99.5: 0.5, \lambda=220 \mathrm{~nm} ; \mathrm{t}_{\mathrm{r}}=16,673 \mathrm{~min}$ and $\left.\mathrm{t}_{\mathrm{r}}=19,700 \mathrm{~min}\right]$.

Figure S1. HPLC traces and UV spectra for racemic 4d

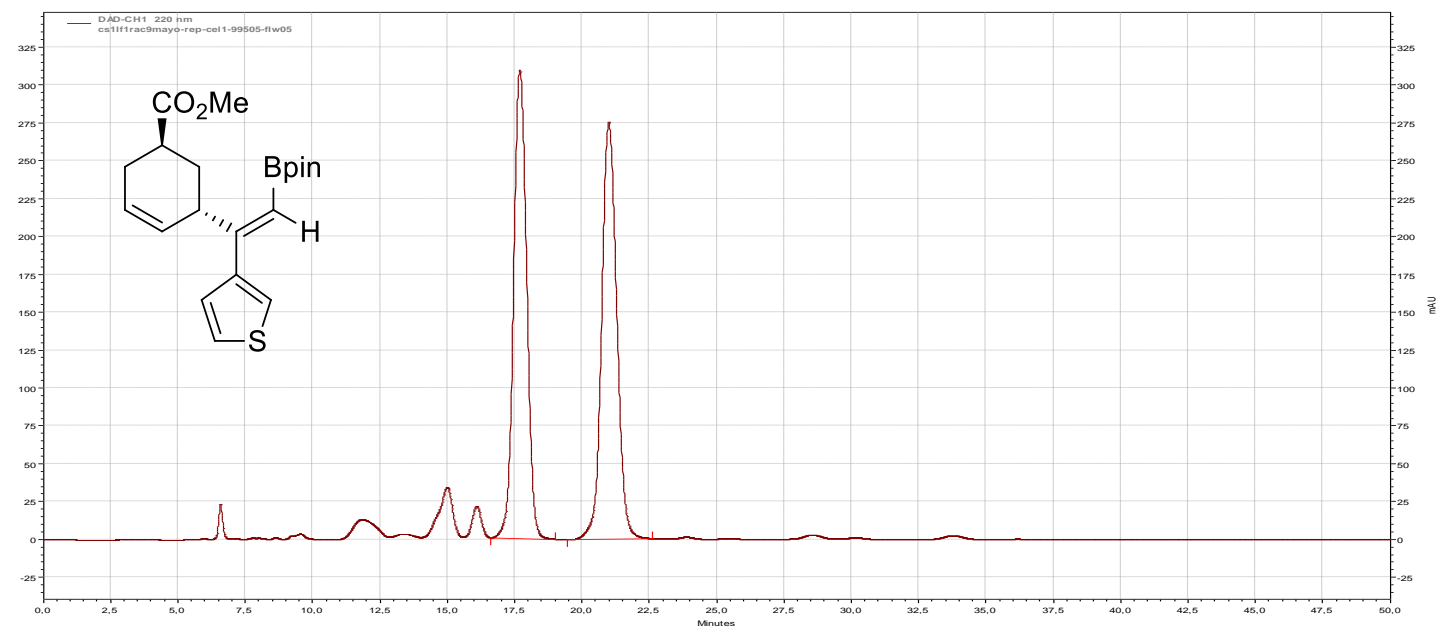

DAD-CH1 220

nm Results

Retention Time

17,867

21,247

Area

106459377

Area $\%$

50,80

2016651

1850805

Height \%

47,86

\begin{tabular}{|r|r|r|r|r|}
\hline Totals & 209572922 & 100,00 & 3867456 & 100,00 \\
\hline
\end{tabular}
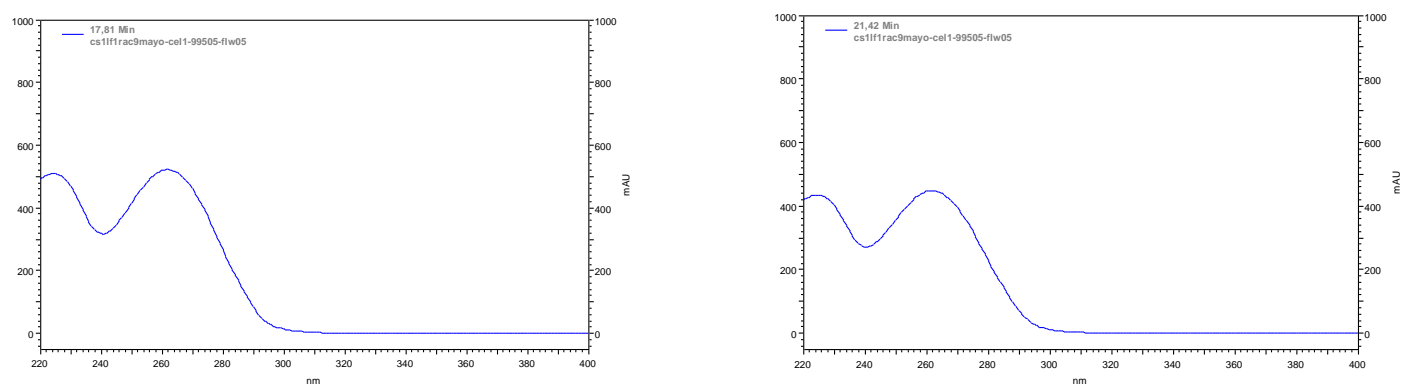
Figure S2. HPLC traces for enantioenriched 4d

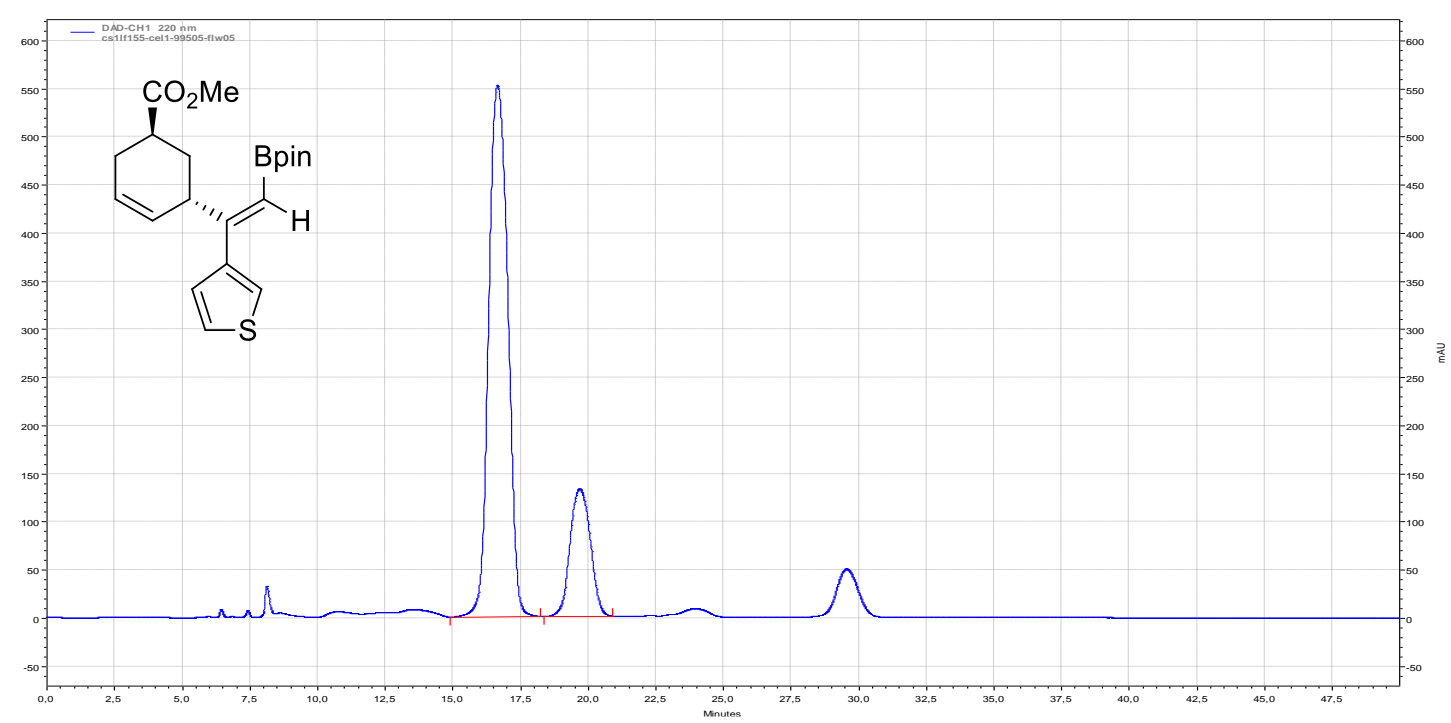

DAD-CH1 220

\section{nm Results}

Retention Time

16,673

19,700

Area

Area \%

Height

Height \%

108519719

79,59

2209897

80,66
19,34

Totals

136347073

100,00

2739658

100,00 
(1R,5S)-N-methoxy- $N$-methyl-5-((E)-1-phenyl-2-(4,4,5,5-tetramethyl-1,3,2-dioxaborolan-2-

yl)vinyl)cyclohex-3-enecarboxamide (4f).<smiles>CON(C)C(=O)C1CC=CC(C(=CC(=O)c2ccccc2)c2ccccc2)C1</smiles>

Obtained from $\mathbf{1} \mathbf{a}, \mathbf{2} \mathbf{b}$ and $\mathrm{B}_{2}(\text { pin })_{2}$ as a yellow oil after column chromatography (hexane/EtOAc 6:1) following general procedure $B$ in $90 \%$ yield and with 77:23 er.

Enantiomeric purity was determined on protodeboronation product $\mathbf{S 1}$ by chiral HPLC analysis [Daicel chiracel IF3 $0.46 \mathrm{~cm} \times 25 \mathrm{~cm}, T_{\text {oven }}=20^{\circ} \mathrm{C}$, flow $=0.5 \mathrm{ml} / \mathrm{min}, \mathrm{Hex} / \mathrm{IPA} 98: 2, \lambda=220 \mathrm{~nm} ; \mathrm{t}_{\mathrm{r}}=30,292 \mathrm{~min}$ and $\left.\mathrm{t}_{\mathrm{r}}=33,844 \mathrm{~min}\right]$.

(1R,5S)-N-methoxy-N-methyl-5-(1-phenylvinyl)cyclohex-3-ene-1-carboxamide (S1)<smiles>C=CC(c1ccccc1)C1C=CCC(C(=O)N(C)OC)C1</smiles>

${ }^{1} \mathrm{H}$ NMR $\left(500 \mathrm{MHz}, \mathrm{CDCl}_{3}\right) \delta 7.44-7.41(\mathrm{~m}, 2 \mathrm{H}), 7.37$ - $7.33(\mathrm{~m}, 2 \mathrm{H}), 7.31$ $7.29(\mathrm{~m}, 1 \mathrm{H}), 6.01-5.96(\mathrm{~m}, 1 \mathrm{H}), 5.82-5.77(\mathrm{~m}, 1 \mathrm{H}), 5.46(\mathrm{~d}, J=1.6 \mathrm{~Hz}$, $1 \mathrm{H}), 5.10(\mathrm{t}, J=1.4 \mathrm{~Hz}, 1 \mathrm{H}), 3.57(\mathrm{~s}, 3 \mathrm{H}), 3.13(\mathrm{~s}, 3 \mathrm{H}), 2.43-2.33(\mathrm{~m}, 2 \mathrm{H})$, $2.21-2.12(\mathrm{~m}, 1 \mathrm{H}), 1.84(\mathrm{td}, J=12.6,6.4 \mathrm{~Hz}, 1 \mathrm{H}), 1.75(\mathrm{~d}, J=13.0 \mathrm{~Hz}, 1 \mathrm{H})$, $0.93(\mathrm{dt}, J=20.0,7.3 \mathrm{~Hz}, 1 \mathrm{H}) .{ }^{13} \mathrm{C}$ NMR $\left(126 \mathrm{MHz}, \mathrm{CDCl}_{3}\right) \delta 169.8,151.3$, 141.6, 128.6, 128.3, 127.9, 127.8, 126.8, 115.2, 61.6, 38.6, 29.5, 28.0. HRMS (APCI) m/z: Calc. for $\mathrm{C}_{17} \mathrm{H}_{22} \mathrm{NO}_{2}[\mathrm{M}+1] 272.1645$, found 272.1642. Optical rotation $[\alpha]^{25}=+20.26(c=0.5$ in $\left.\mathrm{CHCl}_{3}\right)$.

Figure S3. HPLC traces and UV spectra for racemic S1

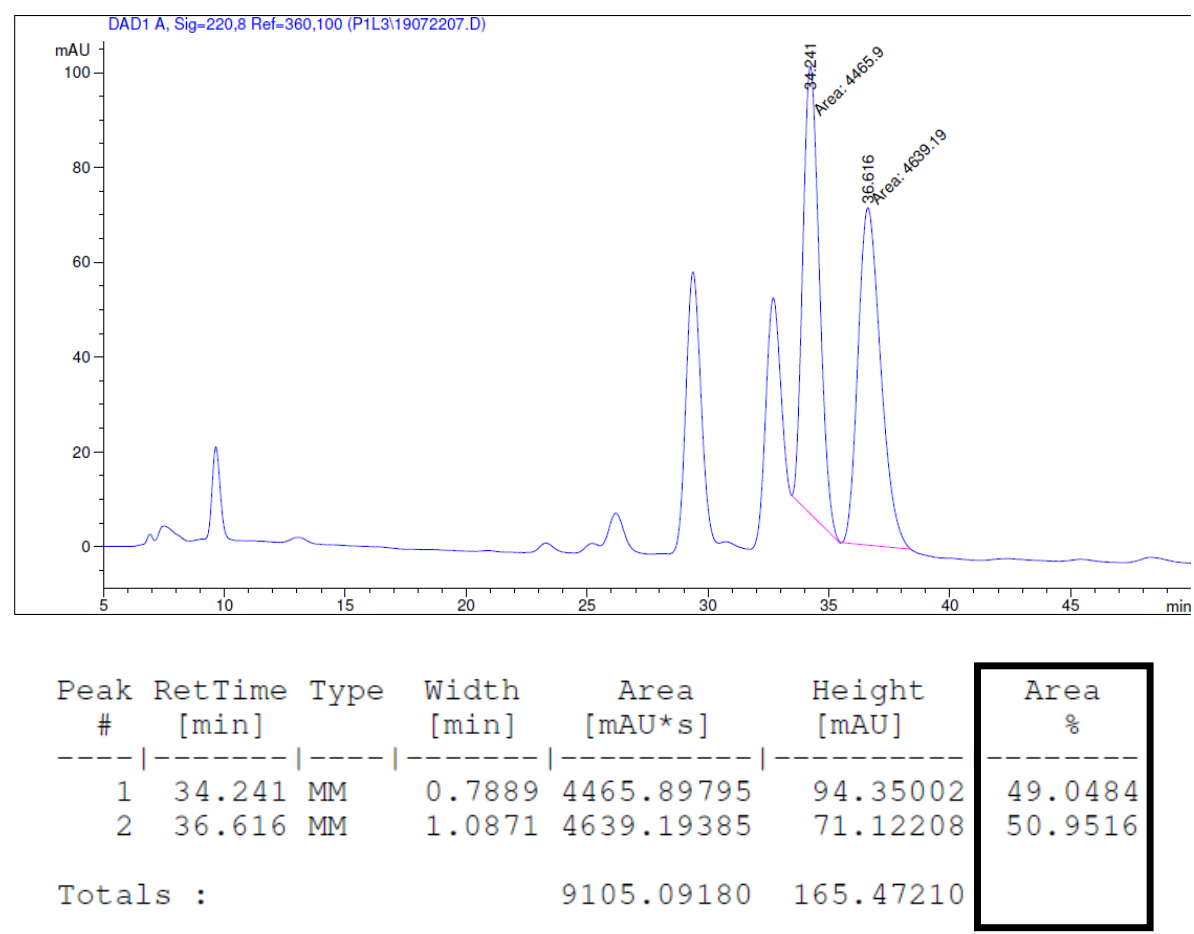

Note: peaks at 29.38 and 32.699 min correspond to Suzuki cross coupling product of $\mathbf{4 f}$ and 1 bromo-4-methylbenzene. 

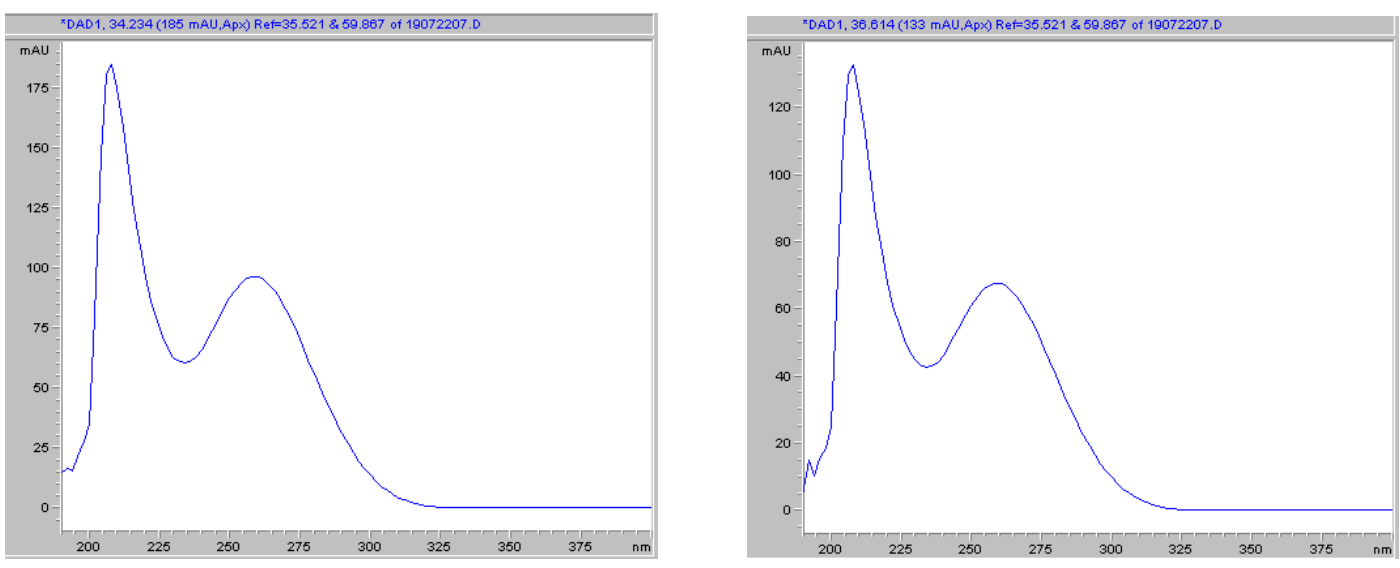

Figure S4. HPLC traces for enantioenriched S1

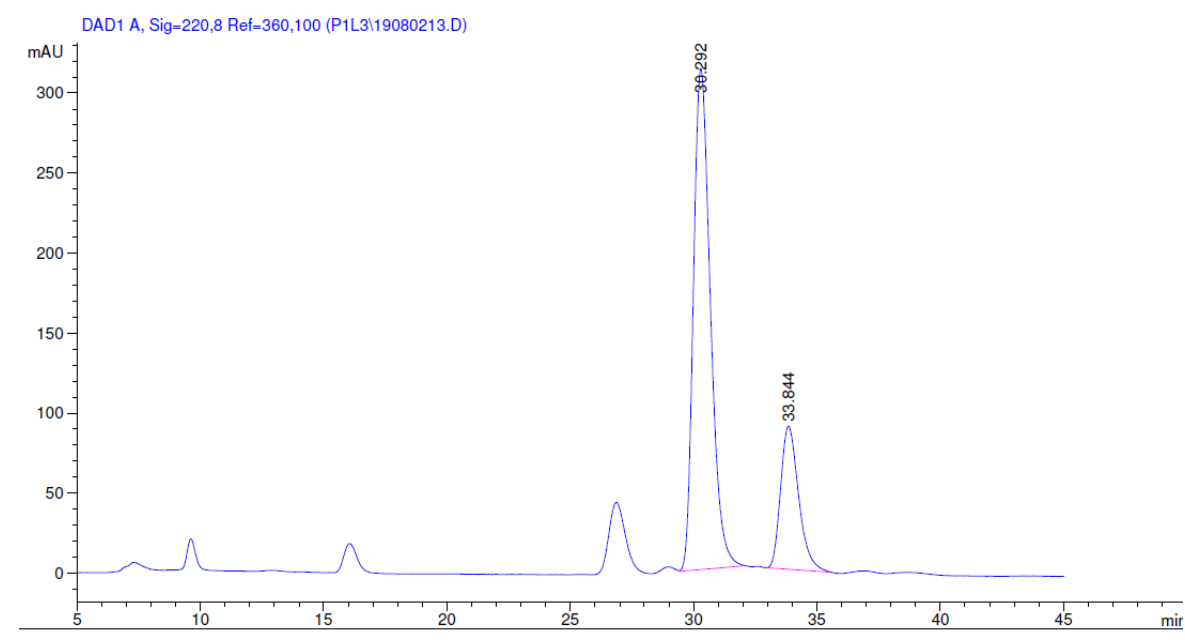

\begin{tabular}{|c|c|c|c|c|c|c|}
\hline $\begin{array}{c}\text { Peak } \\
\quad \#\end{array}$ & $\begin{array}{l}\text { RetTime } \\
\text { [min] }\end{array}$ & Type & $\begin{array}{l}\text { Width } \\
\text { [min] }\end{array}$ & $\begin{array}{c}\text { Area } \\
{\left[\mathrm{mAU}^{*} \mathrm{~s}\right]}\end{array}$ & $\begin{array}{l}\text { Height } \\
\text { [mAU] }\end{array}$ & $\begin{array}{c}\text { Area } \\
\frac{\circ}{0}\end{array}$ \\
\hline $\begin{array}{l}1 \\
2\end{array}$ & $\begin{array}{l}30.292 \\
33.844\end{array}$ & $\begin{array}{l}\text { VB } \\
\text { PB }\end{array}$ & $\begin{array}{l}0.7391 \\
0.7663\end{array}$ & $\begin{array}{l}1.49536 e 4 \\
4541.01025\end{array}$ & $\begin{array}{r}313.14905 \\
89.44154\end{array}$ & $\begin{array}{l}76.7064 \\
23.2936\end{array}$ \\
\hline Tota & 1s : & & & $1.94947 e 4$ & 402.59058 & \\
\hline
\end{tabular}


4,4,5,5-Tetramethyl-2-((E)-2-((1R,3S)-1,2,3,6-tetrahydro-[1,1'-biphenyl]-3-yl)-2-(thiophen-3yl)vinyl)-1,3,2-dioxaborolane (4g).

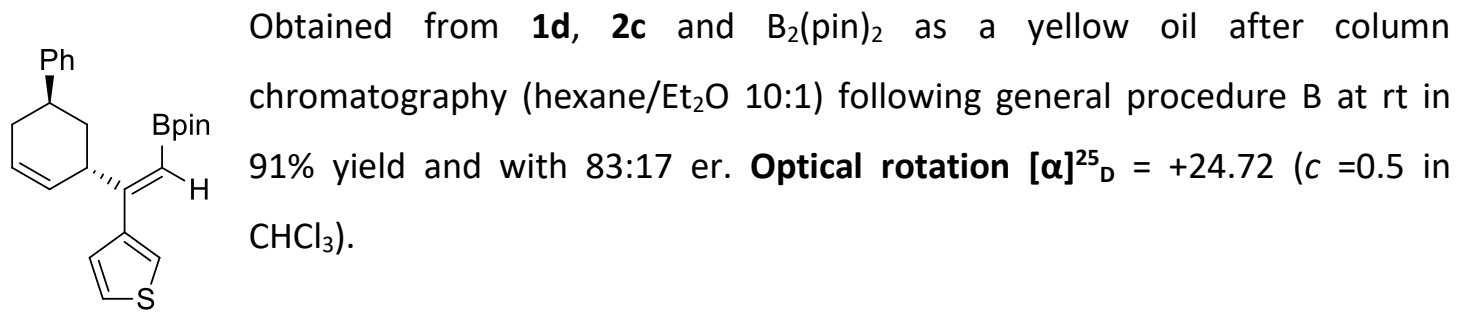

Enantiomeric purity was determined by chiral HPLC analysis [Cellulose1, $\mathrm{T}_{\text {oven }}=40{ }^{\circ} \mathrm{C}$, flow $=$ $0.5 \mathrm{ml} / \mathrm{min}, \mathrm{Hex} / \mathrm{IPA} 99.5: 0.5, \lambda=220 \mathrm{~nm} ; \mathrm{t}_{\mathrm{r}}=22.939 \mathrm{~min}$ and $\left.\mathrm{t}_{\mathrm{r}}=29.983 \mathrm{~min}\right]$.

Figure S5. HPLC traces and UV spectra for racemic $\mathbf{4 g}$

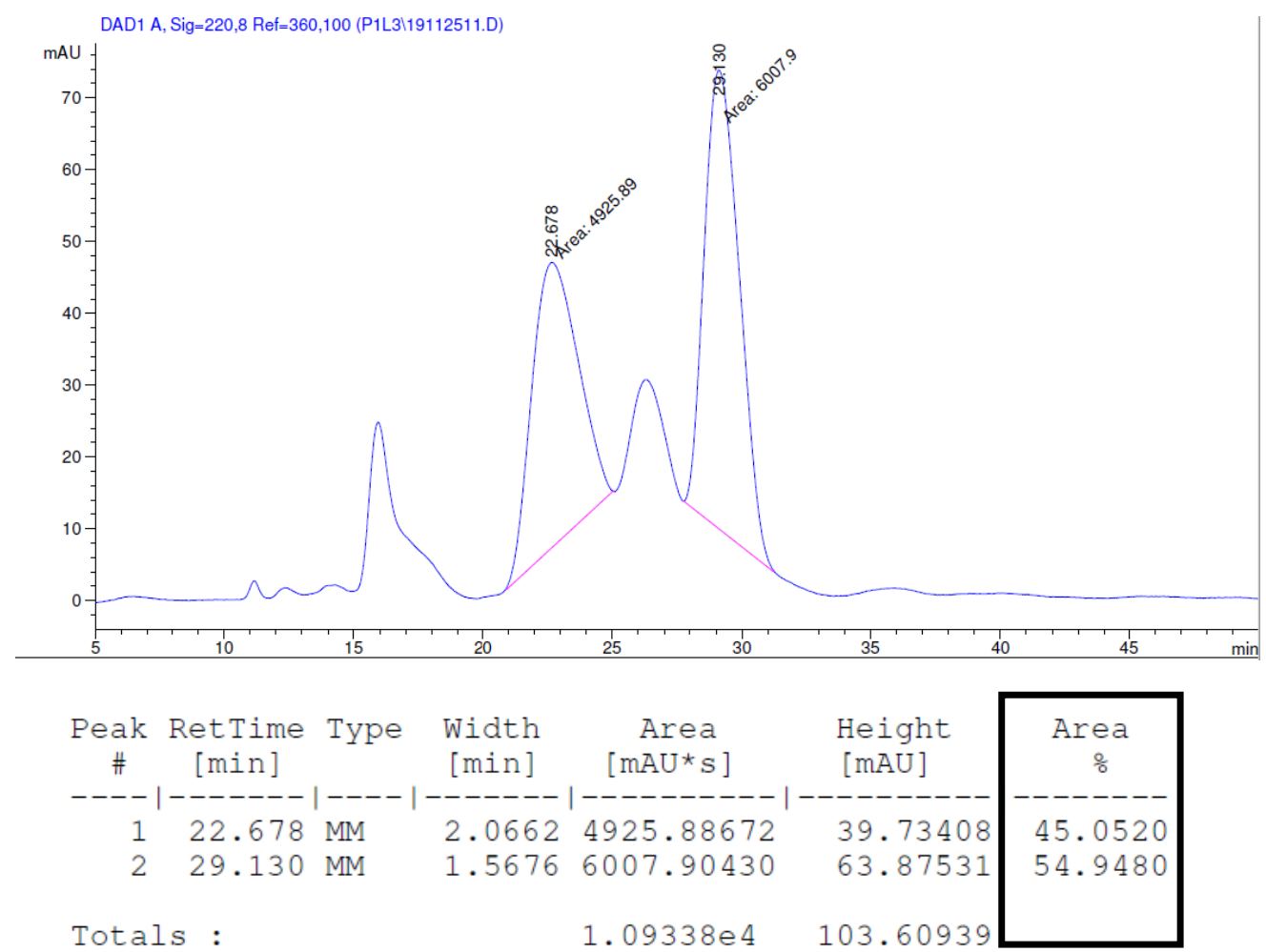

Note: peak at 26.303 min corresponds to the protoboration product $\left(10 \%{ }^{1} \mathrm{H}\right.$ NMR) which could not be removed.

$t_{r}=22.678 \mathrm{~min}$

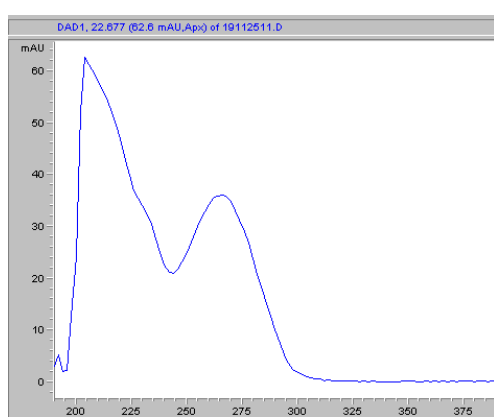

$t_{r}=26.303 \mathrm{~min}$

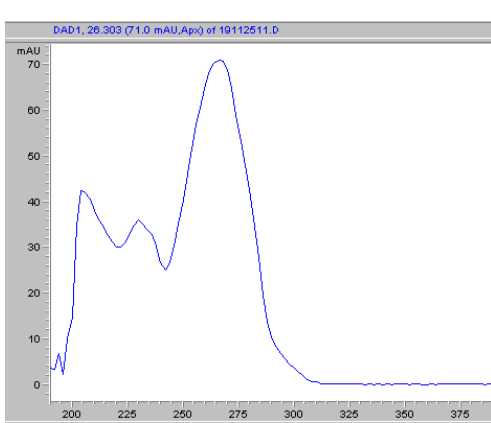

$t_{r}=29.130 \mathrm{~min}$

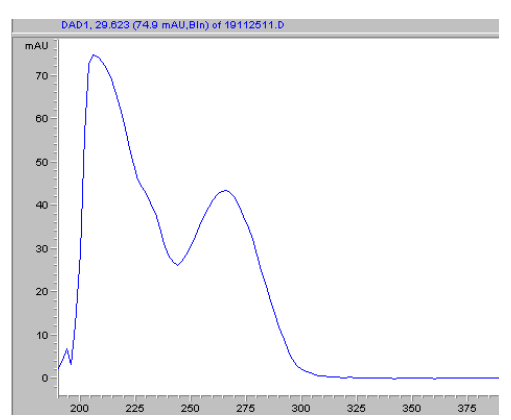


Figure S6. HPLC traces for enantioenriched 4g

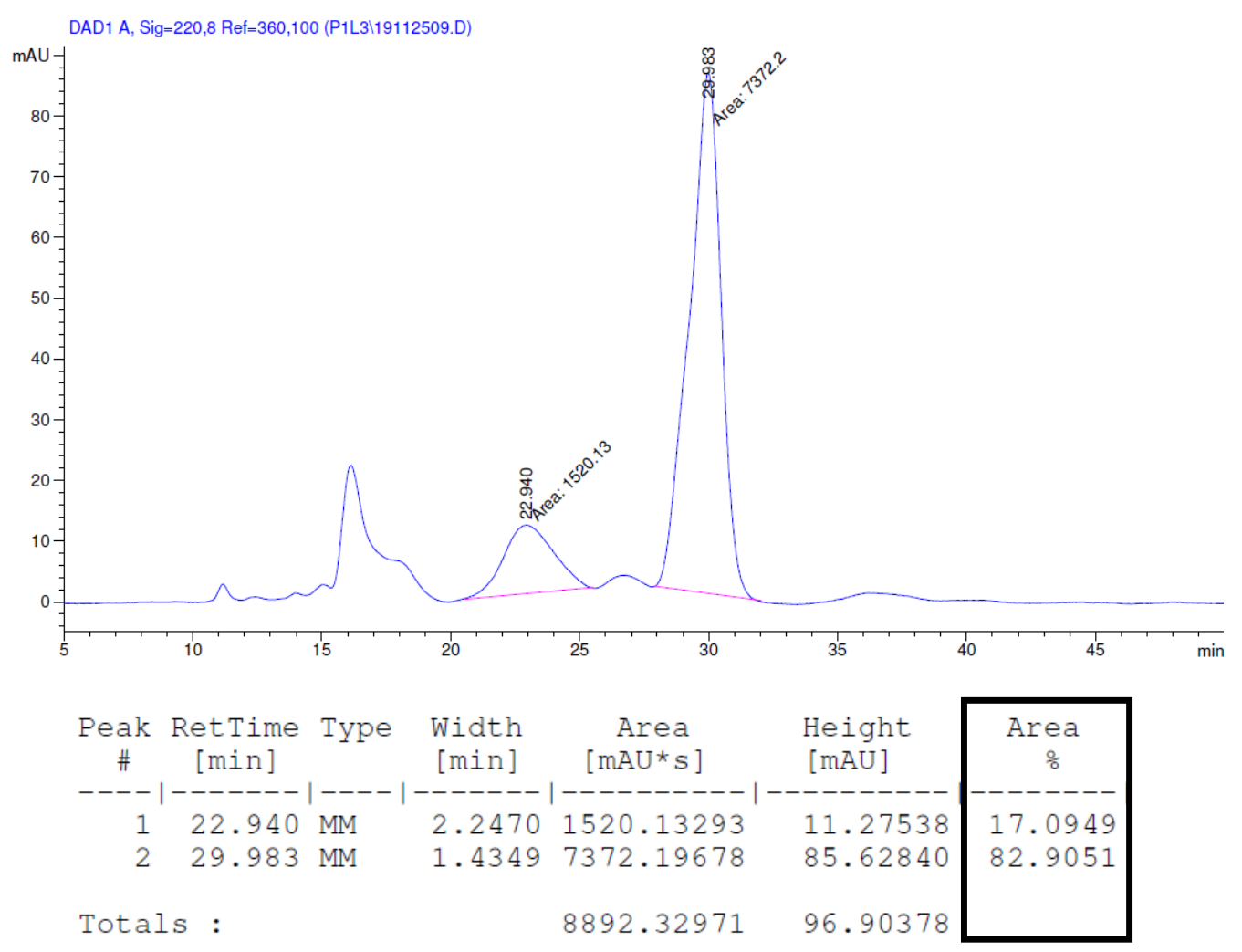




\section{7. ${ }^{1} \mathrm{H}$ and ${ }^{13} \mathrm{C}$ NMR spectra}

Figure S7. 1H NMR spectrum (500 MHz, Chloroform-d) of 4a

$1 \mathrm{H}-\mathrm{NMR}(\mathrm{CDCl}, 500 \mathrm{MHz})$

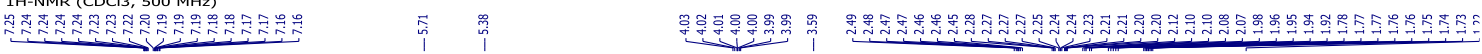<smiles>CC(=O)C1CC=CC(C(=CC(=O)c2ccccc2)c2ccccc2)C1</smiles>

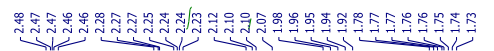
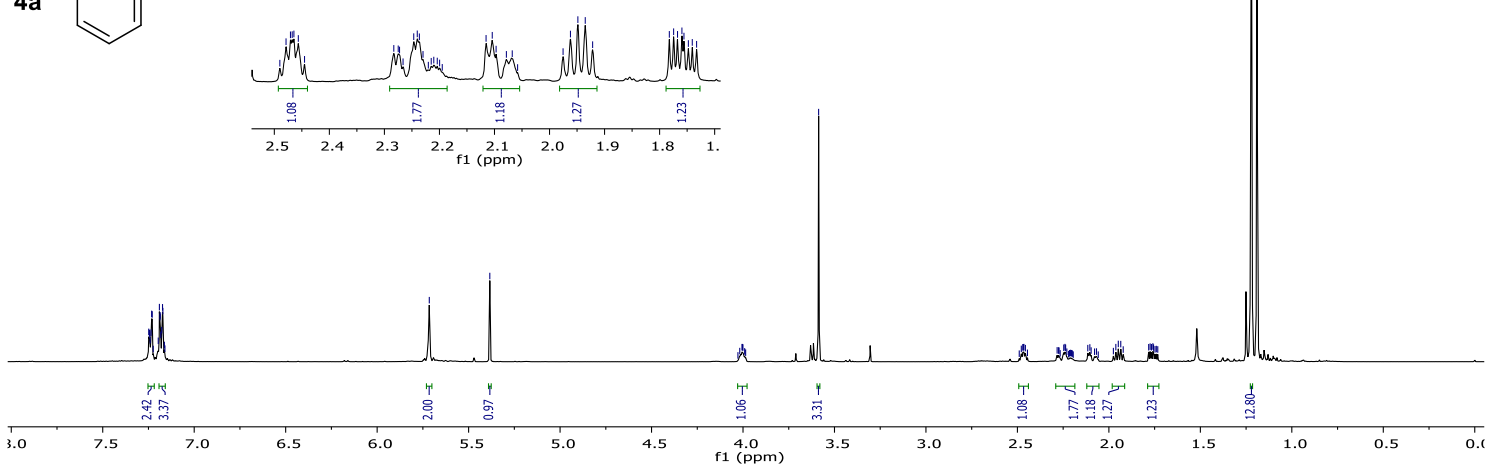

Figure S8. 13C NMR spectrum (126 MHz, Chloroform-d) of 4a

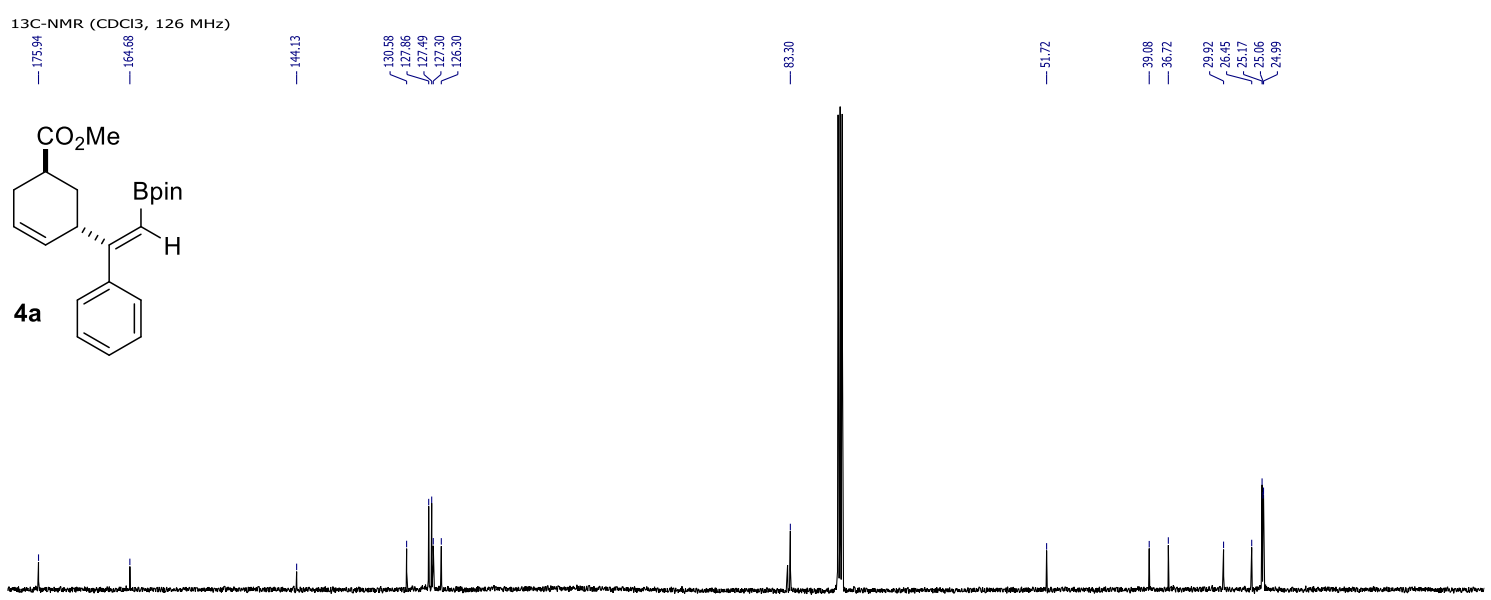


Figure S9. 1H NMR spectrum (500 MHz, Chloroform-d) of $4 \mathbf{b}$

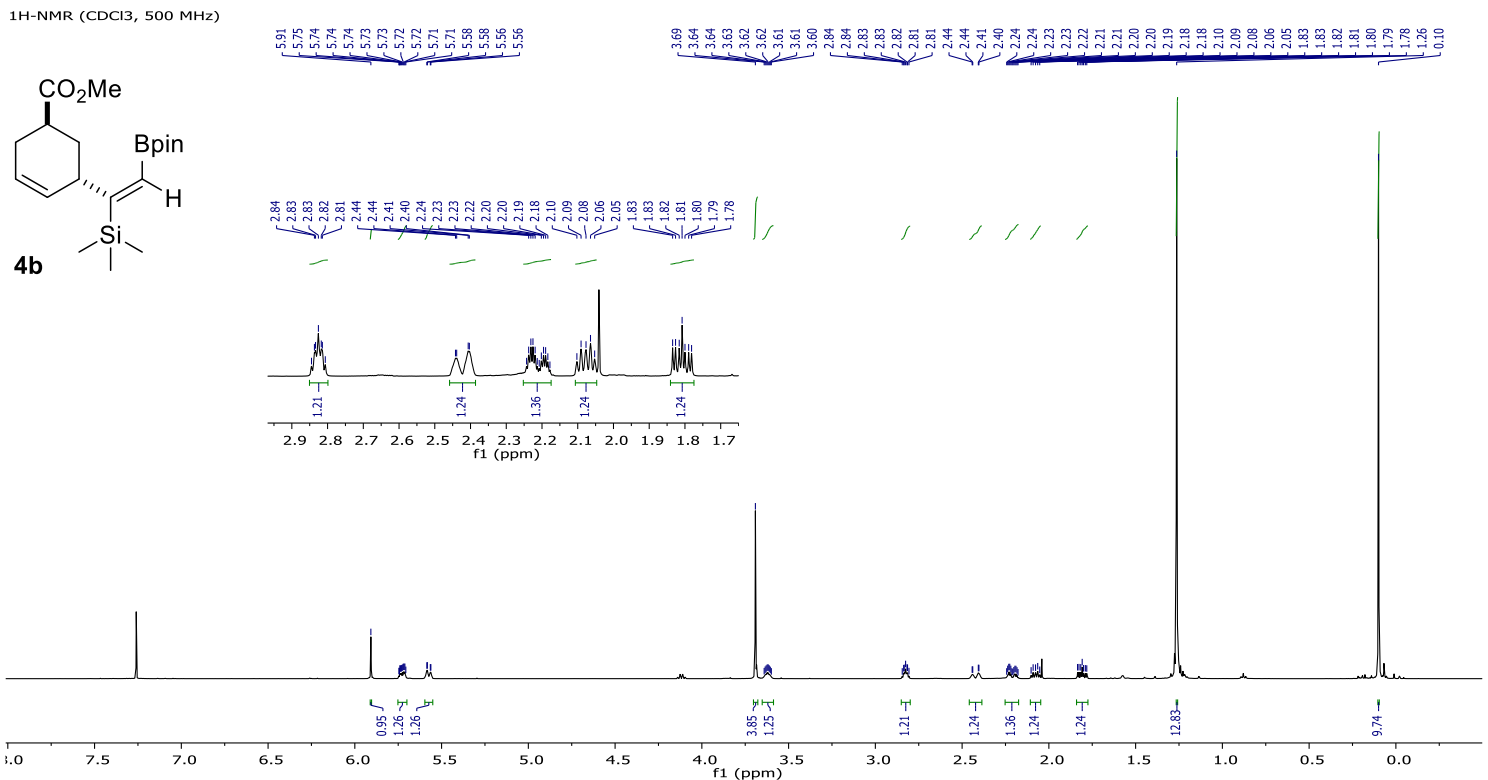

Figure S10. 13C NMR spectrum (126 MHz, Chloroform-d) of 4b
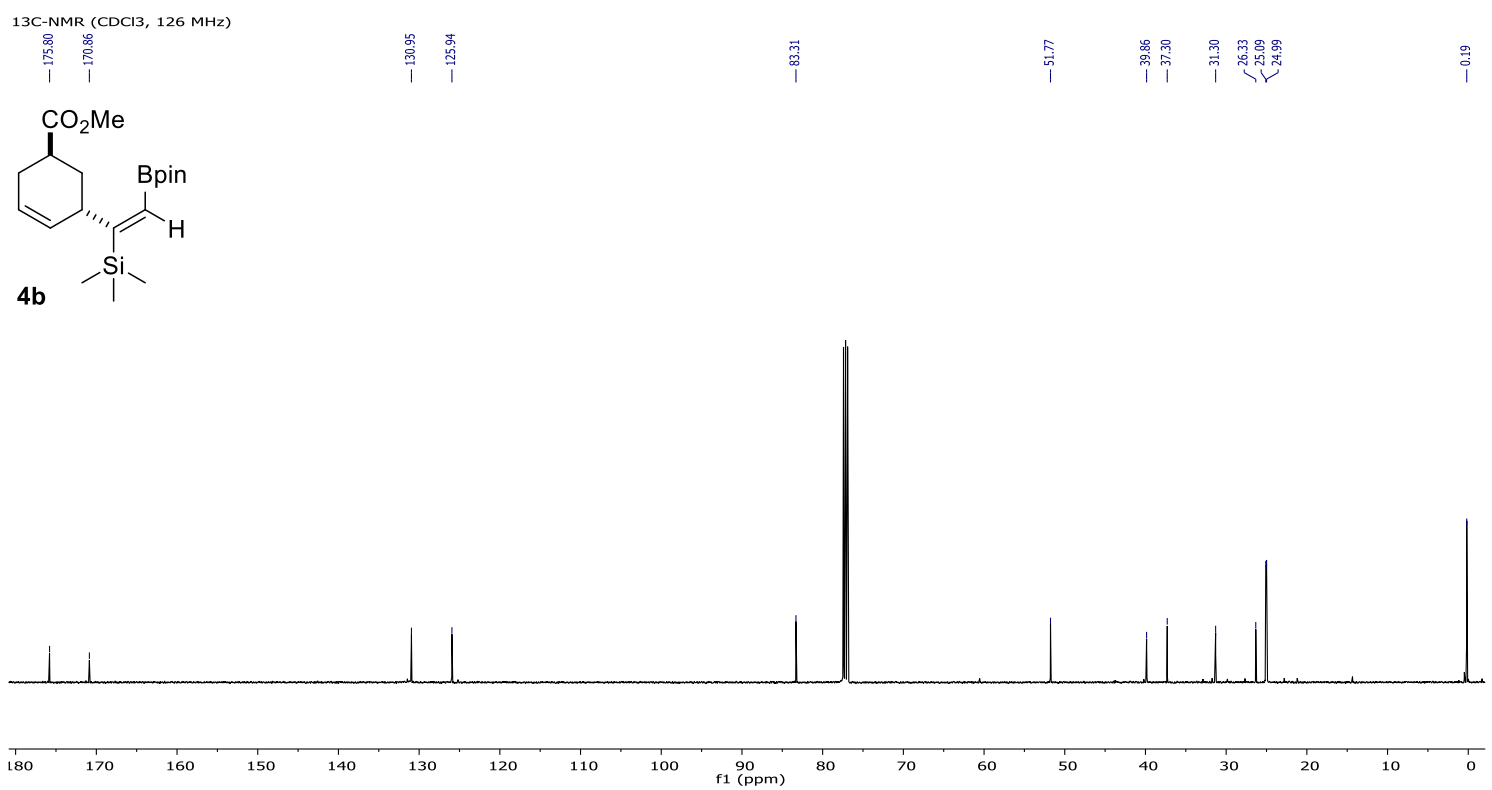
Figure S11. 1H NMR spectrum ( $500 \mathrm{MHz}$, Chloroform-d) of 4c<smiles>CCCCC(=CCc1ccccc1)C1C=CCC(C(C)=O)C1</smiles>
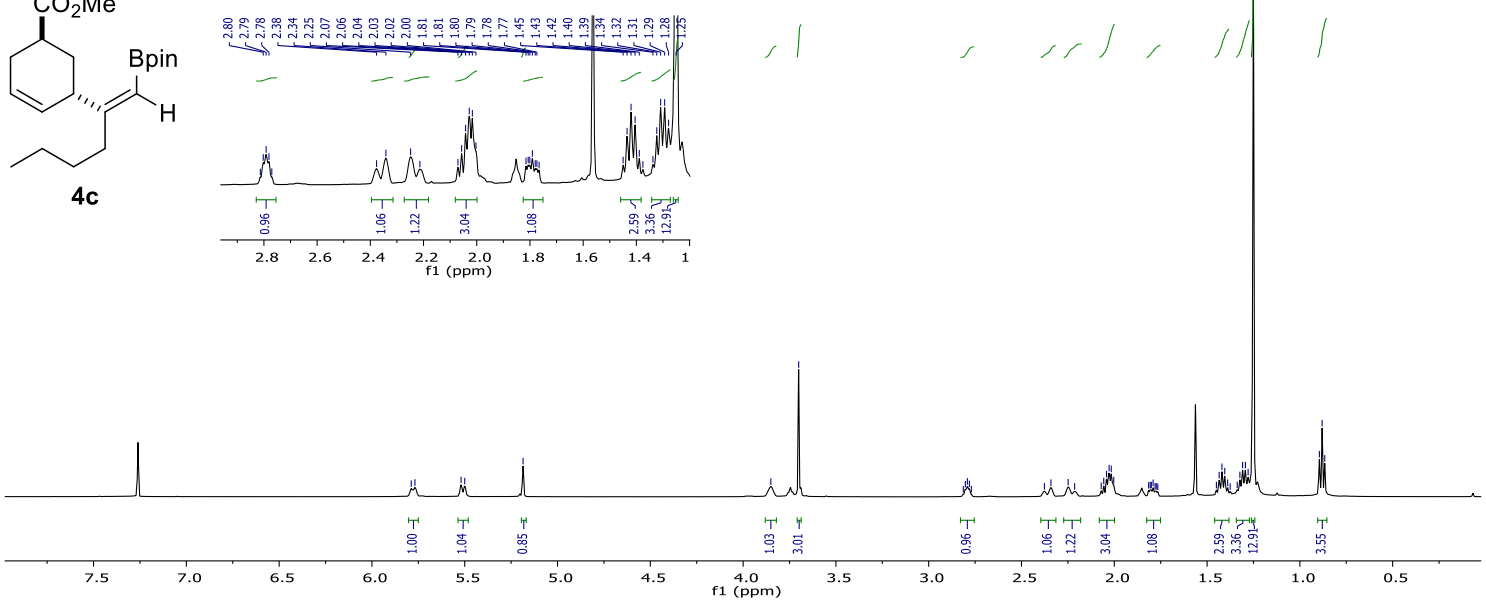

Figure S12. 13C NMR spectrum (126 MHz, Chloroform-d) of 4c

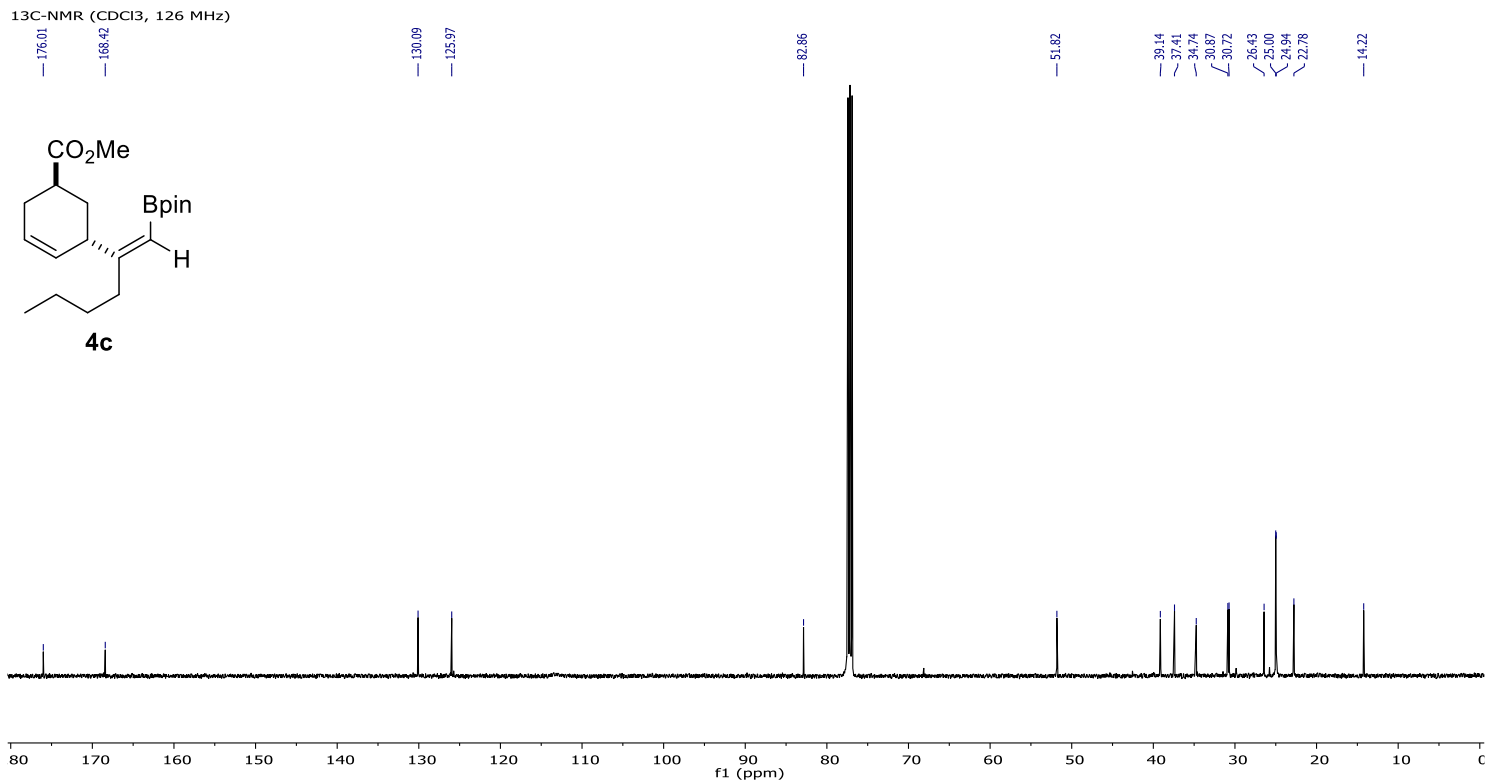


Figure S13. 1H NMR spectrum (500 MHz, Chloroform-d) of 4d

$1 \mathrm{H}-\mathrm{NMR}(\mathrm{CDCl} 3,500 \mathrm{MHz})$

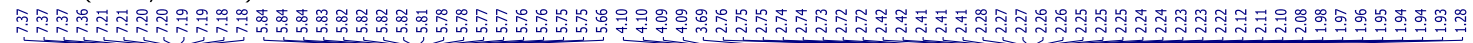
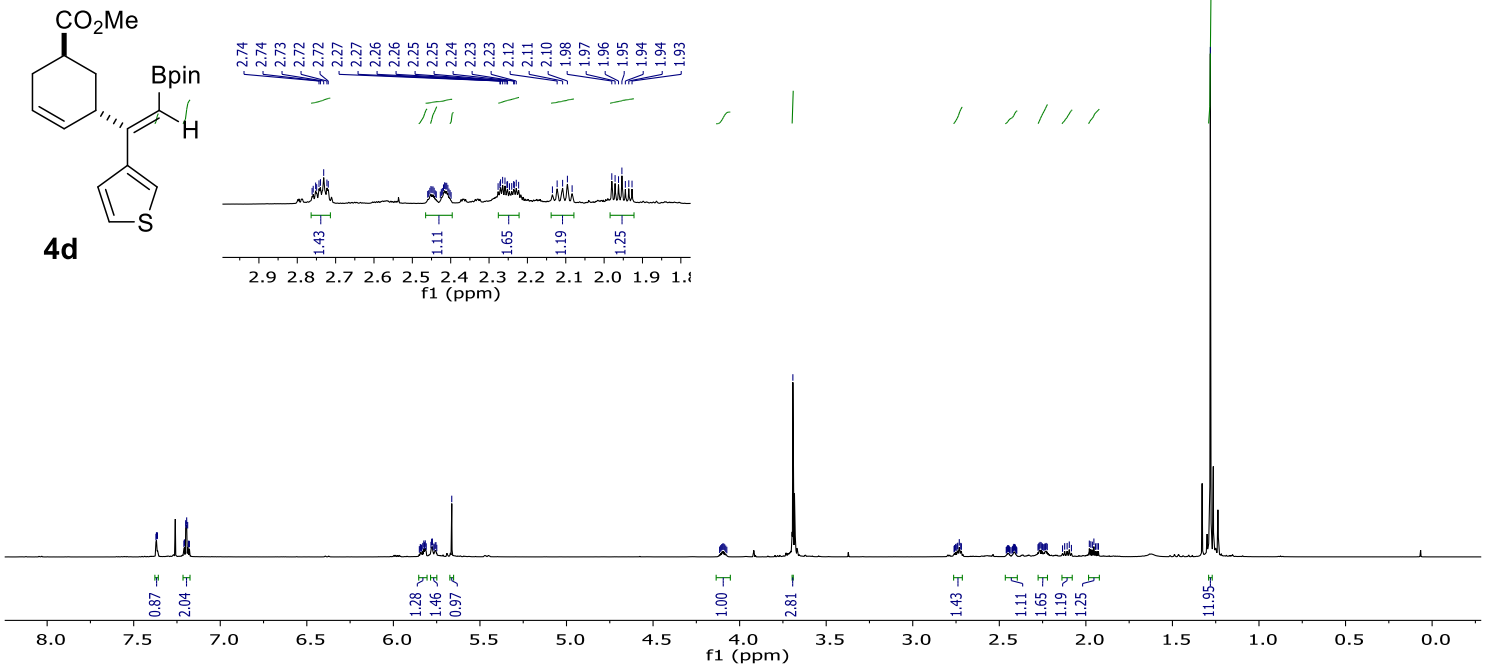

Figure S14. 13C NMR spectrum (75 MHz, Chloroform-d) of 4d

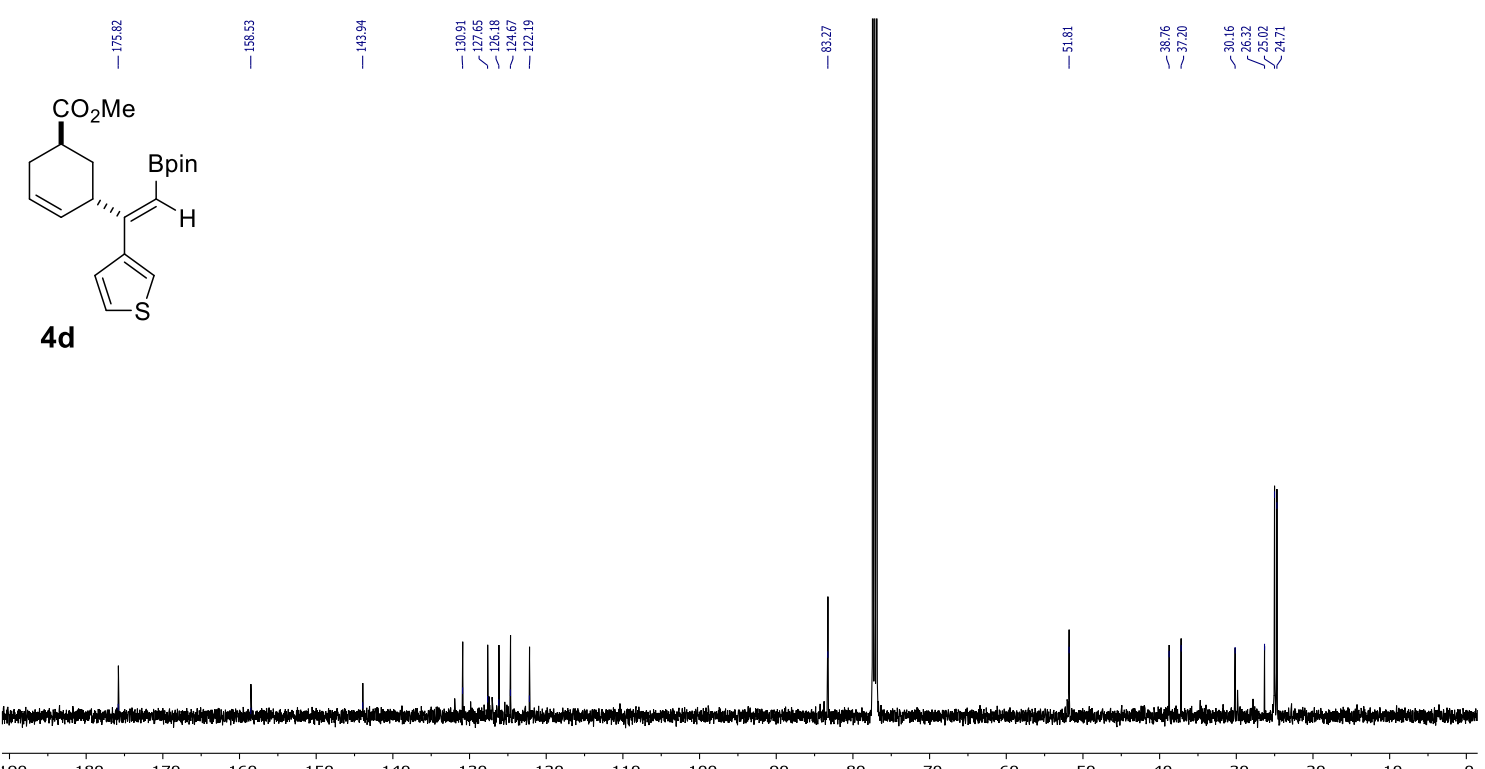

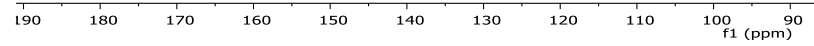


Figure S15. 1H NMR spectrum (500 MHz, Chloroform-d) of cis-4d

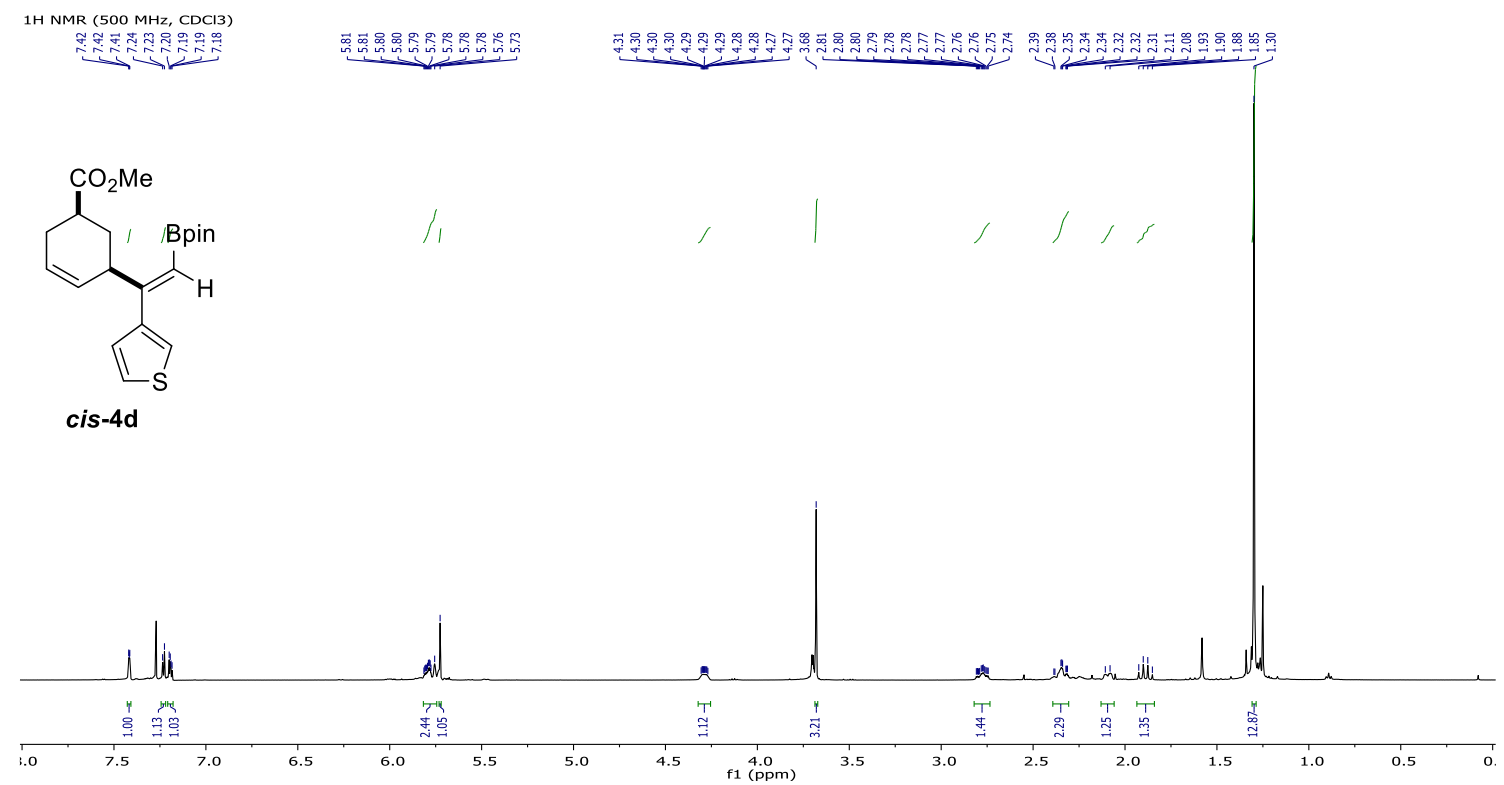

Figure S16. 13C NMR spectrum (126 MHz, Chloroform-d) of cis-4d
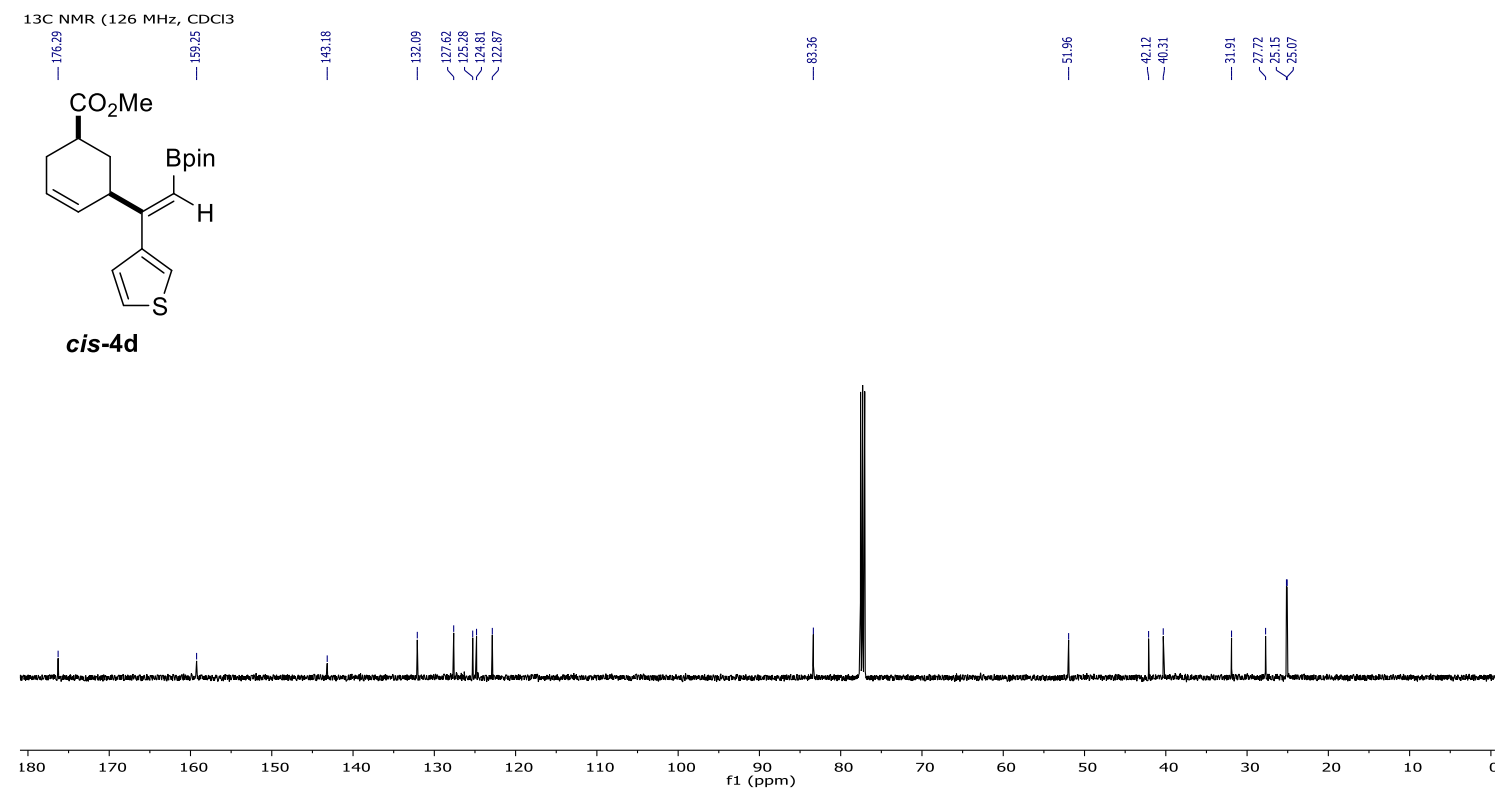
Figure S17. 1H NMR spectrum (500 MHz, Chloroform-d) of 4e<smiles>CC(=O)C1CC=C[C@H](/C(=C(/C)Cc2ccccc2)c2ccccc2)C1</smiles>

$4 \mathrm{e}$

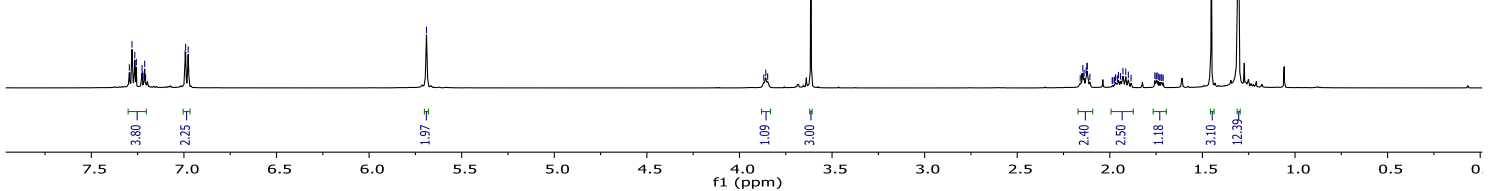

Figure S18. 13C NMR spectrum (126 MHz, Chloroform-d) of 4e
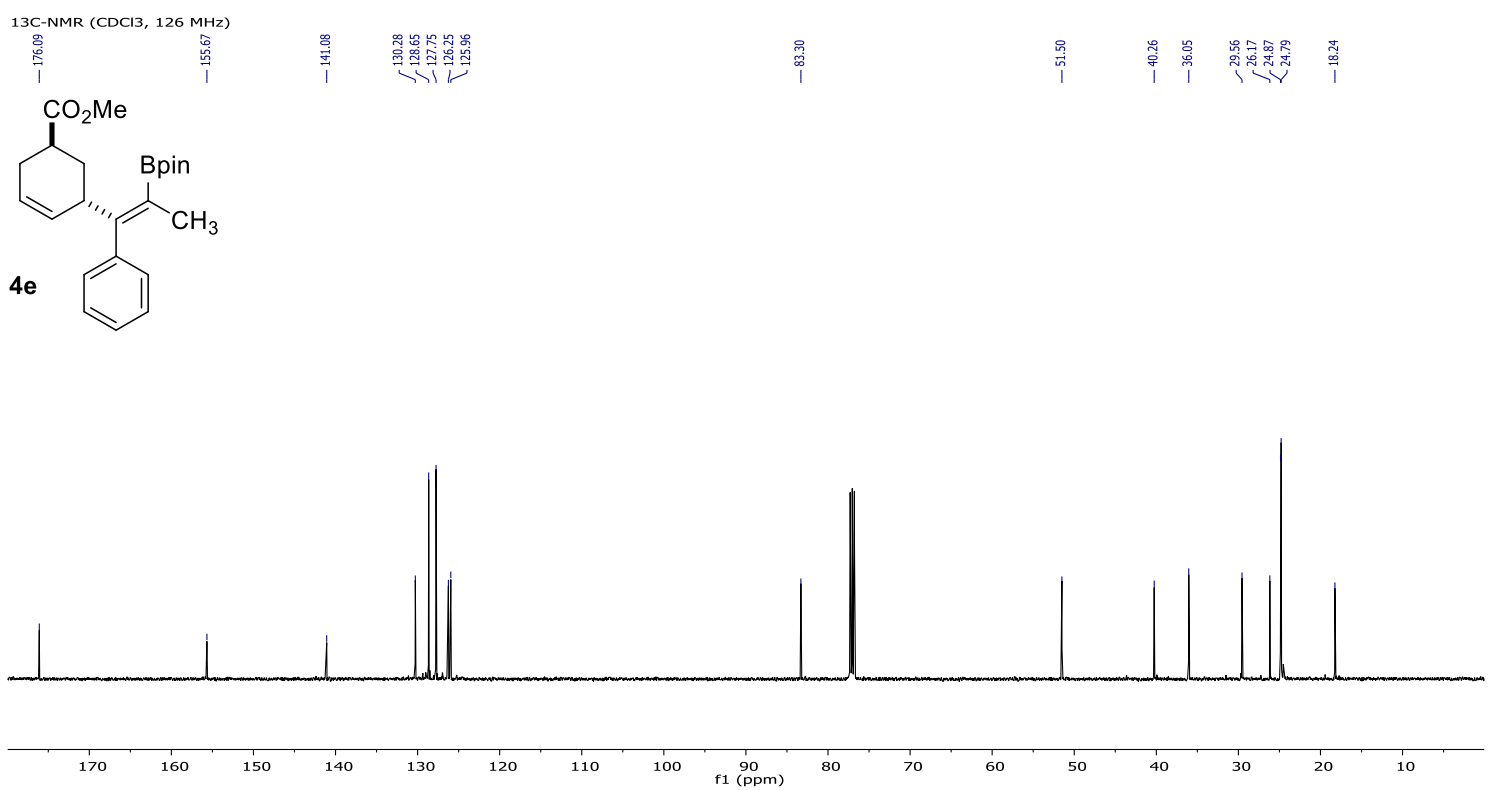
Figure S19. $1 \mathrm{H}$ NMR spectrum (300 MHz, Chloroform-d) of $\mathbf{4 f}$

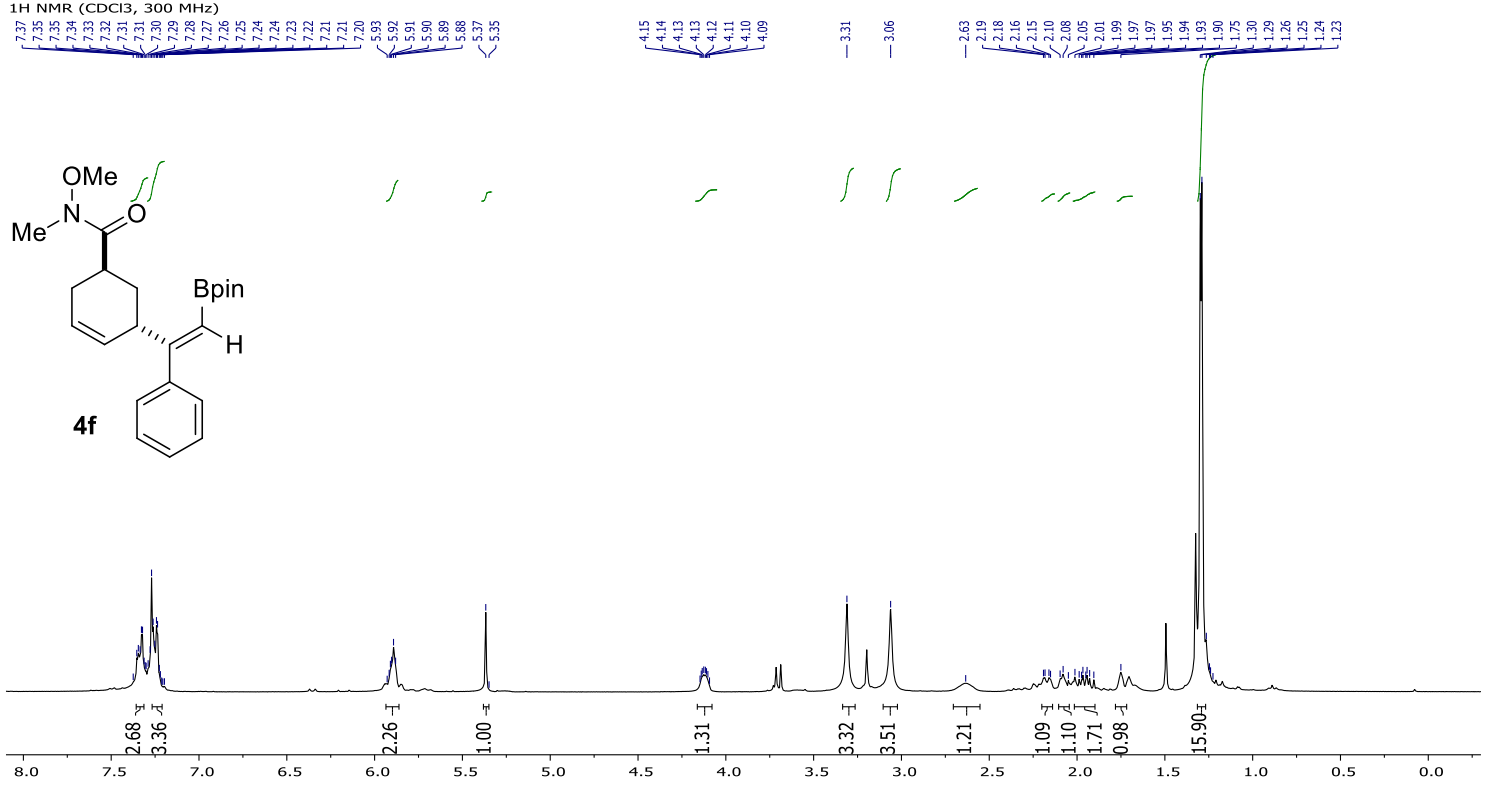

Figure S20. 13C NMR spectrum (75 MHz, Chloroform-d) of $\mathbf{4 f}$
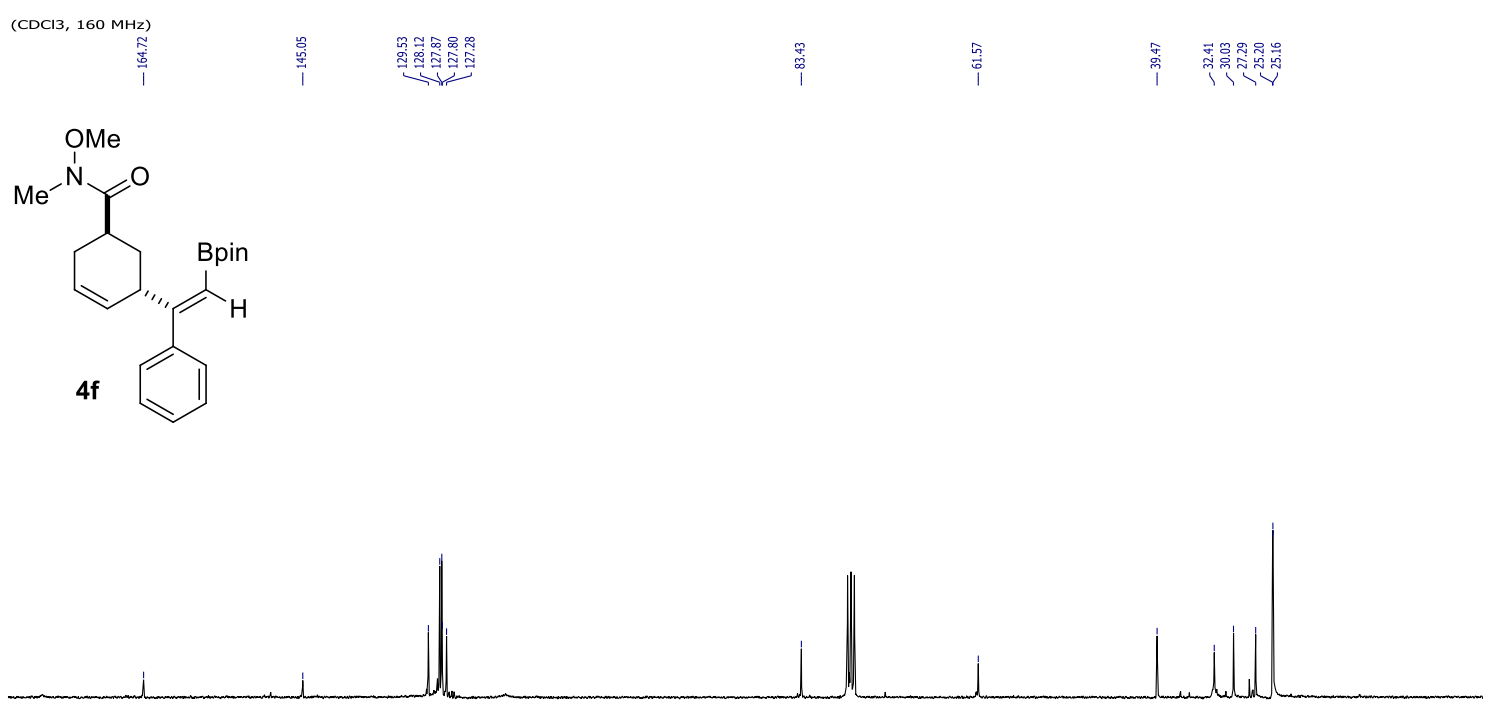

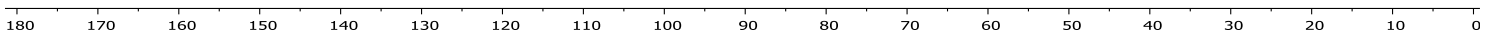


Figure S21. 1H NMR spectrum (300 MHz, Chloroform-d) of $\mathbf{4 g}$

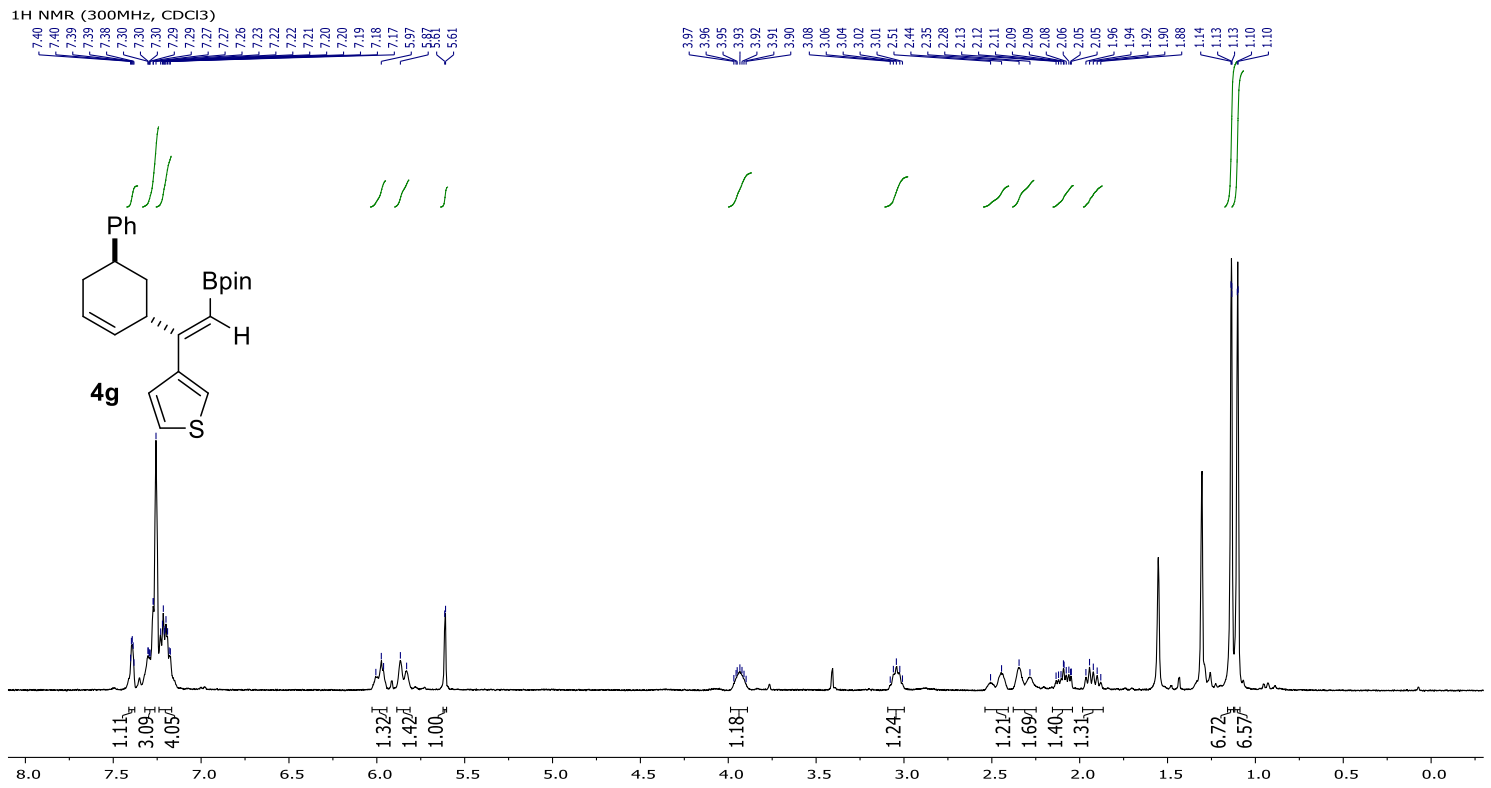

Figure S22. 13C NMR spectrum (126 MHz, Chloroform-d) of 4g

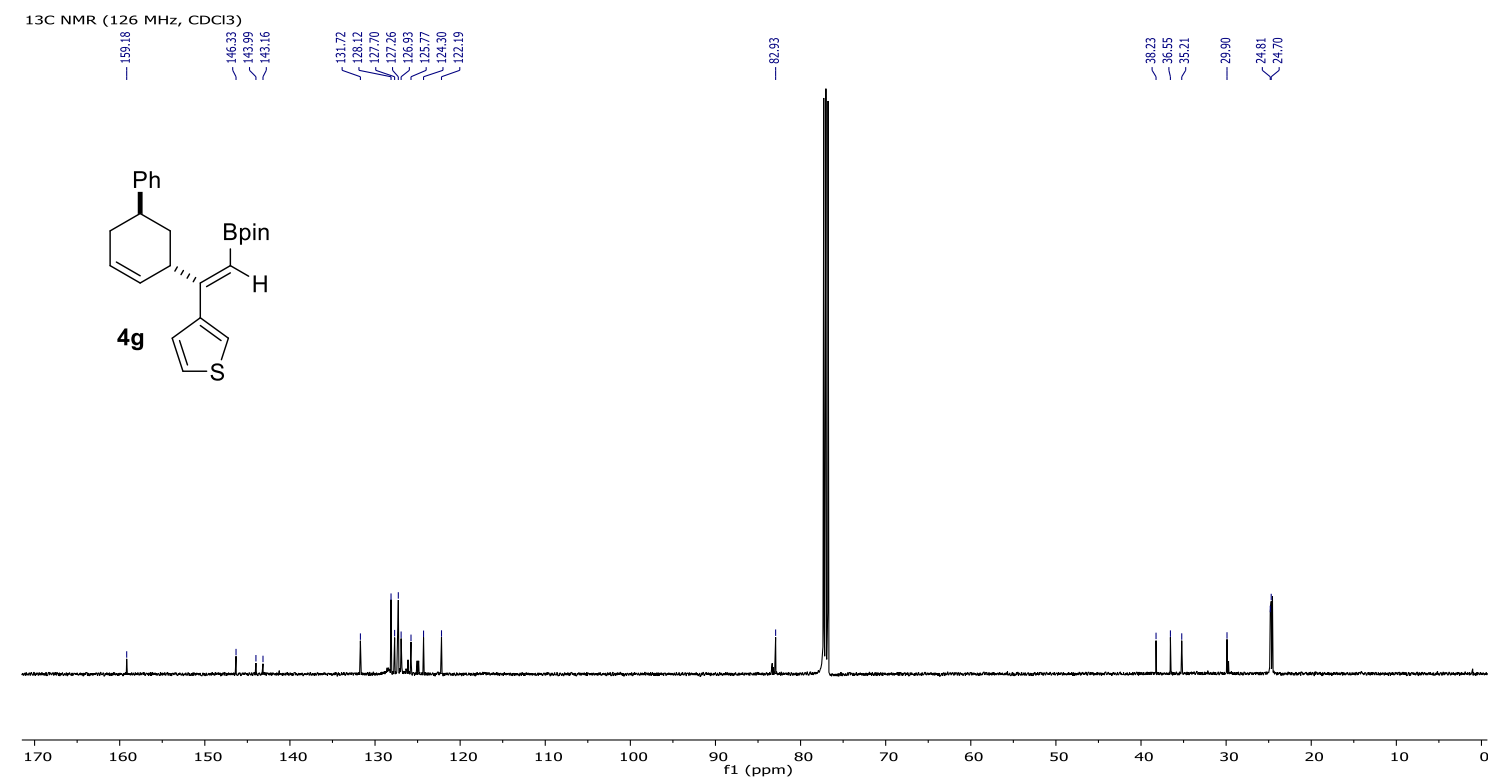


Figure S23. $1 \mathrm{H}$ NMR spectrum ( $500 \mathrm{MHz}$, Chloroform-d) of $\mathbf{4 h}$<smiles>C#CC=C(c1ccccc1)[C@H]1C=CCC(c2ccccc2)C1</smiles>
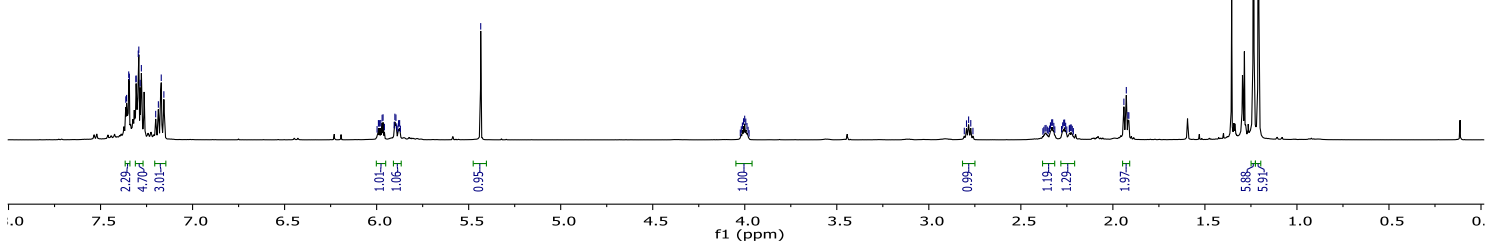

Figure S24. 13C NMR spectrum (126 MHz, Chloroform-d) of 4 h
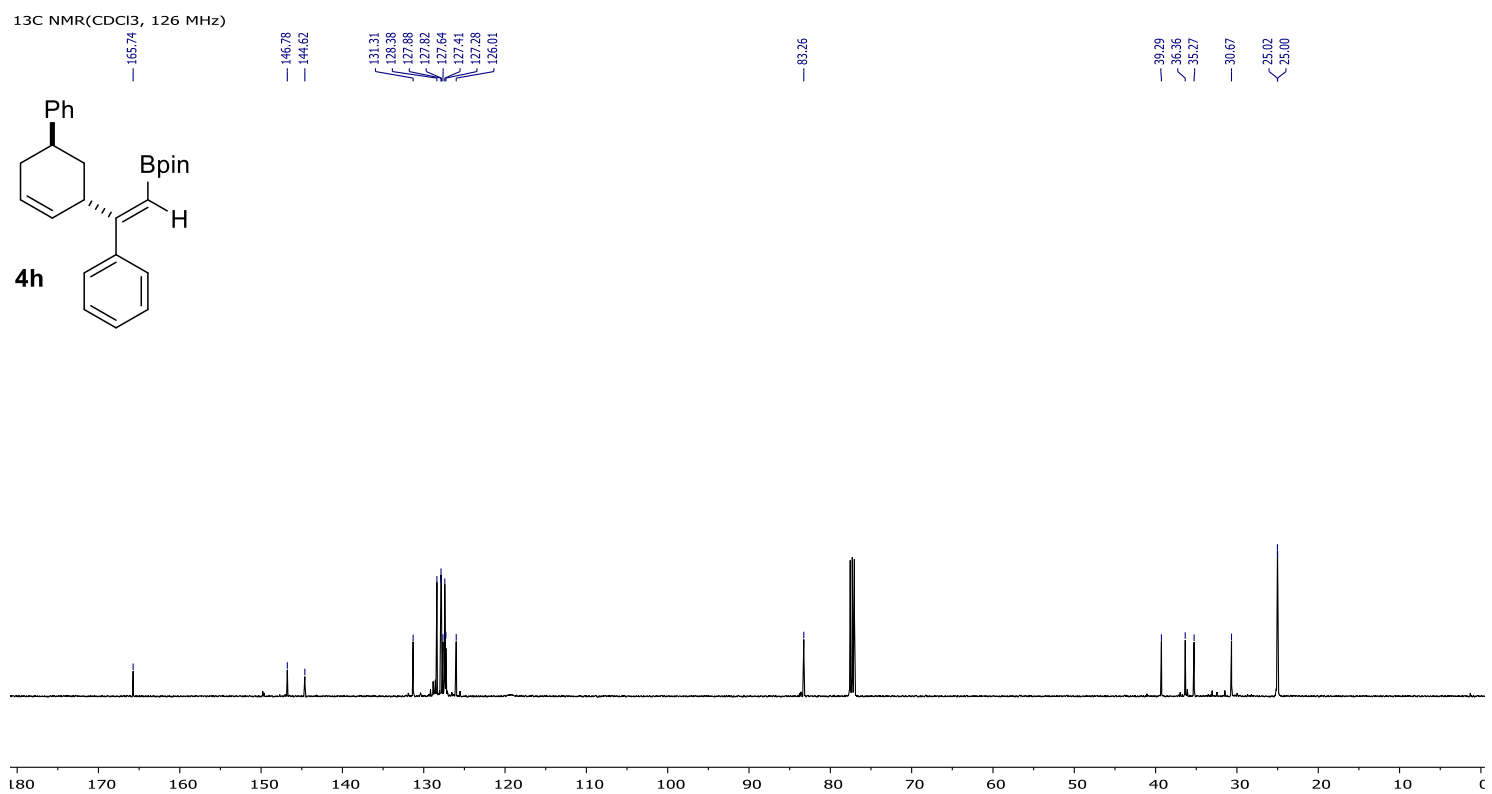
Figure S25. 1H NMR spectrum (500 MHz, Chloroform-d) of S1

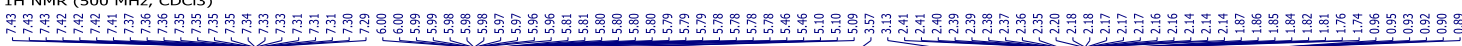<smiles>C#CC=CC1C=CCC(C(=O)N(C)OC)C1</smiles>

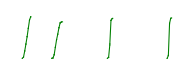

S1

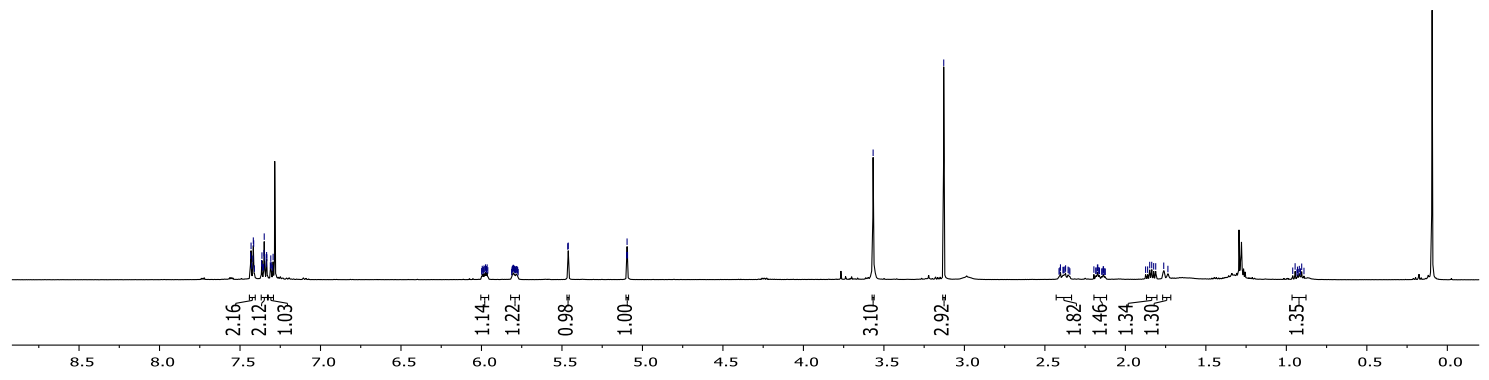

Figure S26. 13C NMR spectrum (126 MHz, Chloroform-d) of S1

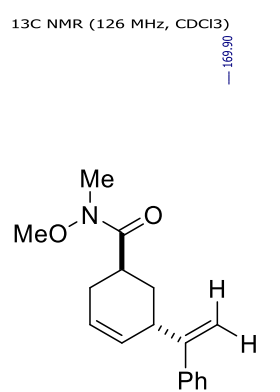

S1
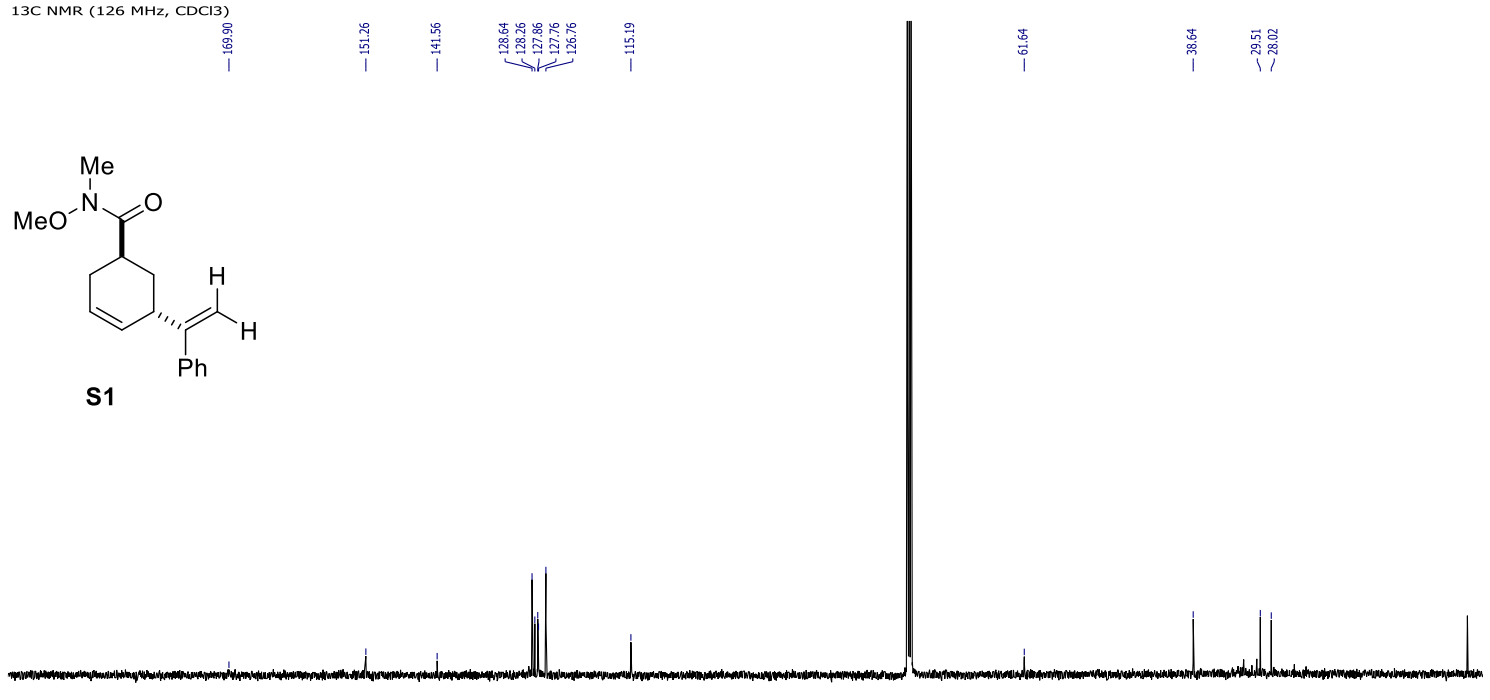

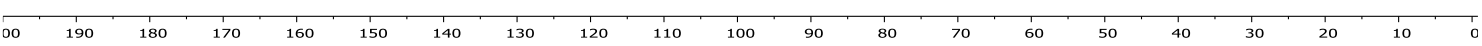


8. Stereochemical determination of compounds trans-4a and cis-4d by NOE experiments

Figure S27. NOE NMR spectrum of trans-4a

trans-4a
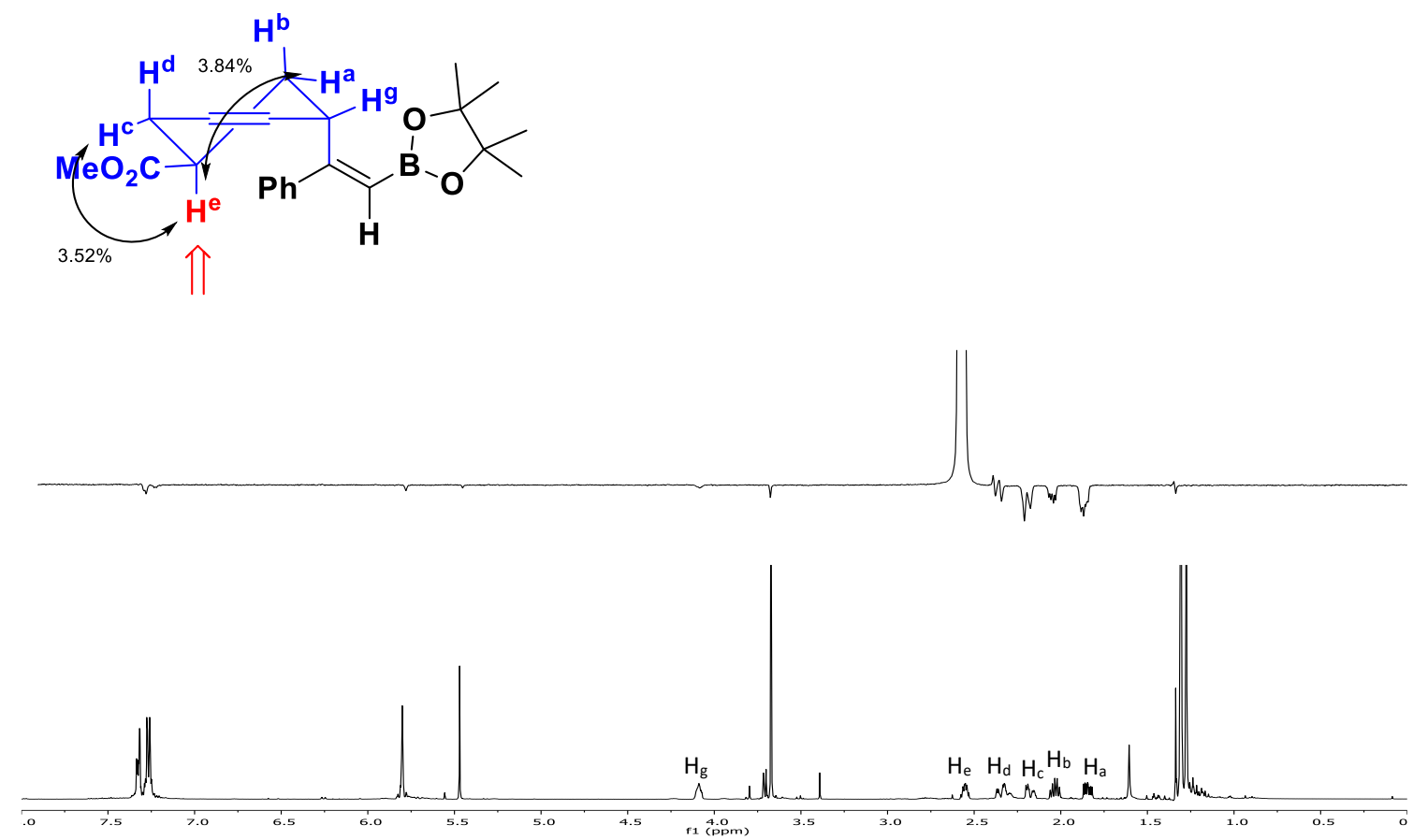

Figure S28. NOE NMR spectrum of cis-4d
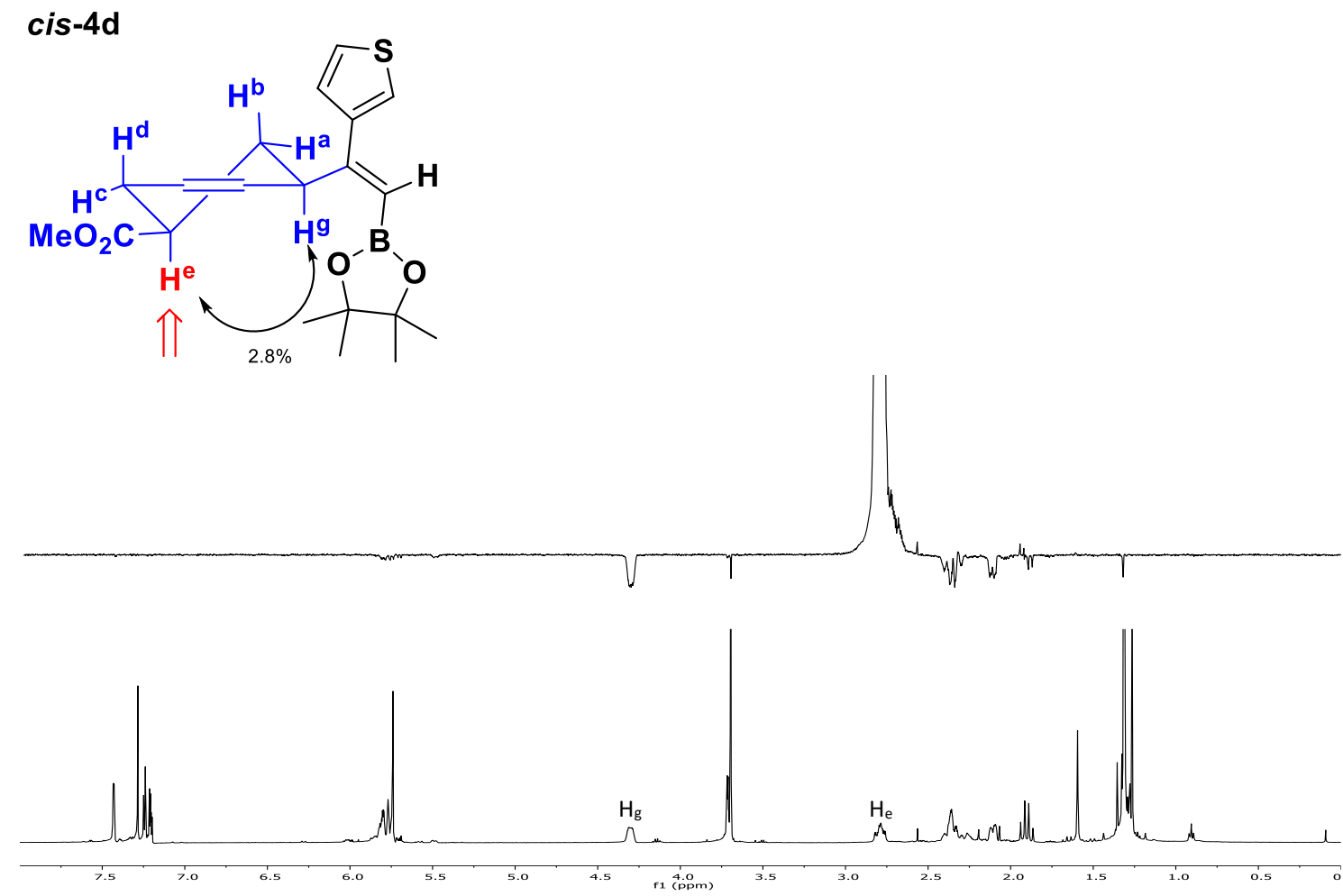


\section{References}

[1] Misale, A.; Niyomchon, S.; Luparia, M.; Maulide, N. Asymmetric Palladium-Catalyzed Allylic Alkylation Using Dialkylzinc Reagents: A Remarkable Ligand Effect. Angew. Chem. Int. Ed. 2014, $53,7068$.

[2] Murahashi, S.; Taniguchi, Y.; Imada, Y.; Tanigawa, Y. Palladium (0)-catalyzed azidation of allyl esters. Selective synthesis of allyl azides, primary allylamines, and related compounds. J. Org. Chem, 1989, 54, 3292.

[3] Koschker, P.; Kähny, M.; Breit, B. Enantioselective Redox-Neutral Rh-Catalyzed Coupling of Terminal Alkynes with Carboxylic Acids Toward Branched Allylic Esters. J. Am. Chem. Soc. 2015, $137,3131$.

[4] Li, W.; Waldkirch, J. P.; Zhang, X. Chiral $C_{2}$-Symmetric Ligands with 1,4-Dioxane Backbone Derived from Tartrates: Syntheses and Applications in Asymmetric Hydrogenation. J. Org. Chem. 2002, 67, 7618.

[5] a) Nagao, Y.; Ikeda, T.; Inoue, T.; Yagi, M.; Shiro, M.; Fujita E. J. Org. Chem. 1985, 50, 4072;

b) McKinstry, L.; Livinghouse, T. An efficient procedure for the synthesis of C-chiral bisphosphines. Tetrahedron, 1995, 51, 7655; c) Huang, K.; Zhang, X.; Emge, T. J.; Hou, G.; Cao, B.; Zhang, X. Design and synthesis of a novel three-hindered quadrant bisphosphine ligand and its application in asymmetric hydrogenation. Chem. Commun. 2010, 46, 8555. 